\title{
Proposal for a loophole-free test of the Bell inequalities
}

\author{
Edward S. Fry, ${ }^{*}$ Thomas Walther, and Shifang $\mathrm{Li}^{\dagger}$ \\ Physics Department, Texas A\&M University, College Station, Texas 77843-4242
}

(Received 22 May 1995)

\begin{abstract}
A proposal for an experimental realization of Bohm's spin- $\frac{1}{2}$ particle version of the Einstein-Podolsky-Rosen experiment is described. Two ${ }^{199} \mathrm{Hg}$ atoms, each with nuclear spin $\frac{1}{2}$, are produced in an entangled state with total nuclear spin zero. Such a state is obtained by dissociation of dimers of the ${ }^{199} \mathrm{Hg}_{2}$ isotopomer using a spectroscopically selective stimulated Raman process. The measurement of nuclear spin correlations between the two atoms in this entangled state is achieved by detection of the atoms using a spin state selective two-photon excitation-ionization scheme. The experiment will not only close the detector efficiency loophole, but in addition will permit enforcement of the locality condition.
\end{abstract}

PACS number(s): 03.65.Bz; 32.80.Fb

\section{INTRODUCTION}

In the early years of the development of quantum mechanics (QM), there was great exhilaration because of its capability of providing accurate statistical predictions. However, in those early days (and even today) there were many who expressed a great deal of concern about the interpretation of quantum mechanics for single microscopic systems. Foremost among them was Albert Einstein, who together with Boris Podolsky and Nathan Rosen (generally referred to as EPR) wrote a paper in 1935 in which they expressed their concern that quantum mechanics was an incomplete theory [1]. Presumably, additional parameters, for which the term "hidden variables" (HV) was coined, would be required in order to restore completeness to the theory. However, since the results of experiments are statistical data, no experiments were immediately obvious and the debate centered on philosophical considerations until 1964.

In that year John Bell published the proof that any hiddenvariable theory satisfying a physically reasonable condition of locality will yield statistical predictions that must satisfy restrictions for certain correlated phenomena [2]. These restrictions have been derived for various situations and in various forms over the years; all are generally referred to as Bell inequalities. Furthermore Bell demonstrated that quantum mechanics yields statistical predictions that can violate these restrictions. Thus for the first time experimental tests were conceivable.

In 1969 Clauser et al. introduced auxiliary assumptions to make physically realizable experiments possible with existing technology [3]. Several, involving polarization correlations between two photons in an atomic cascade, were then initiated [4]. The first [5], third [6], and fourth [7,8] gave results in agreement with QM and clearly violated Bell inequalities. Signals observed in the fourth were larger $(\approx 1.0$ coincidence/sec) than in previous experiments, and systematics could be more thoroughly examined.

In the ensuing years other experiments were performed.

\footnotetext{
*FAX: (409) 845 2590. Electronic address: fry@phys.tamu.edu

${ }^{\dagger}$ Present address: Department of Physics, University of California at Berkeley, Berkeley, California 94720.
}

In 1982 Aspect, Grangier, and Roger used two channel polarizers and achieved extremely high statistical accuracy [9]. Shortly thereafter, they used time-varying analyzers to change the polarizer settings in a quasiperiodic way [10]. This did not rigorously enforce the locality condition, but was an important advance and has been the only successful progress in this direction. Experiments in recent years have involved correlations between the pair of photons produced by down-conversion in a nonlinear crystal [11-13].

All of these experiments required an auxiliary assumption since they involved photons in or near the visible spectrum and employed detectors whose efficiencies were typically $<20 \%$. An inequality that requires no auxiliary assumptions and is therefore called a strong Bell inequality was obtained by Clauser and Horne in 1974 [4,14]; it is especially important because it is formulated in terms consistent with a physically realizable experiment. However, the quantummechanical predictions will only violate this inequality for very high detector efficiencies. Strong Bell inequalities have not yet been tested experimentally.

The advent of solid-state avalanche photodiodes provides a high detection efficiency that could be sufficient for tests of strong Bell inequalities with photons in or near the visible. Such an experiment has been recently described by Kwiat et al. [15].

In this paper we will describe a different type of experimental test of a strong Bell inequality. This experiment also permits enforcement of the locality condition. We will first give a short overview of the experimental concept, then we will briefly discuss the theoretical background, and finally we will present some of the requisite experimental details.

\section{EXPERIMENTAL OVERVIEW}

An overview of the experiment is shown in Fig. 1. Instead of photon pairs, this experiment involves measurements of the correlations between angular-momentum components of two atoms (nuclei) of the isotope ${ }^{199} \mathrm{Hg}[16,17]$. The correlated ${ }^{199} \mathrm{Hg}$ atoms are produced by dissociation of ${ }^{199} \mathrm{Hg}_{2}$ dimers via stimulated Raman excitation to a dissociating state of their $X^{1} \Sigma_{g}^{+}$ground state. The total electron and the total nuclear spin angular momenta are both zero in the initial rotational state of the mercury dimers, and are not 


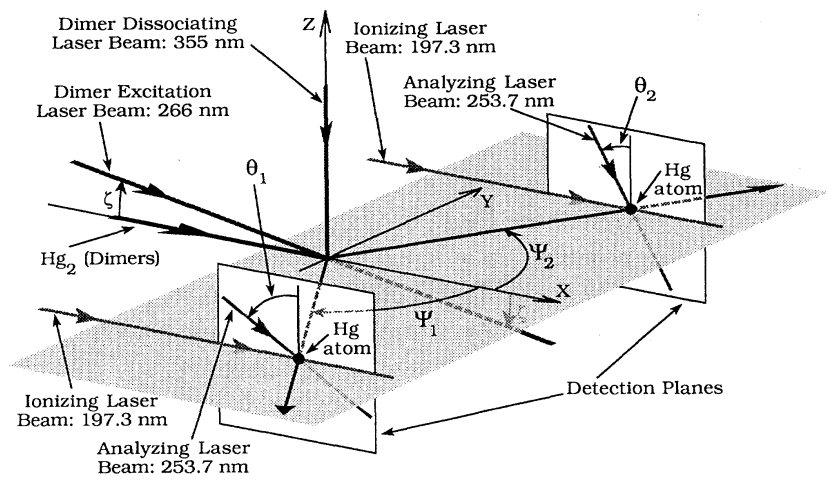

FIG. 1. Schematic of the experiment showing the direction of the mercury dimer beam together with a pair of the dissociated atoms and their respective detection planes. The relative directions of the various laser beams are also shown. Details are discussed throughout the text.

changed in the dissociation process. The two resulting mercury atoms are in ${ }^{1} S_{0}$ ground states. Because of the nuclear spin $I=\frac{1}{2}$ of ${ }^{199} \mathrm{Hg}$, each ground-state atom has total angular momentum $F=\frac{1}{2}$. Consequently we will be observing correlations between components of the spin of two spatially separated spin- $\frac{1}{2}$ particles; it is a direct experimental realization of Bohm's Gedankenexperiment. The component of angular momentum (nuclear spin) in any given direction is measured by orienting excitation laser beams in that direction and using polarization-selective excitation of one of the Zeeman sublevels. Atoms with only one component of angular momentum, $M_{F}= \pm \frac{1}{2}$, are excited. They are then detected $(\approx 100 \%$ efficiency) by photoionization via an autoionizing state.

Rates for simultaneous detection (coincidence rates) of the two atoms at their respective detectors are measured for components of the angular momentum in the directions $\theta_{1}$ and $\theta_{2}$ (i.e., the directions of the excitation laser beams). A set of four angles can be chosen that give a maximum violation of the strong Bell inequality. This experiment also lays the foundation for an experiment that enforces locality since one can stochastically choose the directions of the excitation laser beams on the nanosecond time scale.

\section{THEORETICAL BACKGROUND}

Consider a ${ }^{199} \mathrm{Hg}_{2}$ dimer in an even- $J$ rotational state of the $X^{1} \Sigma_{g}^{+}$ground state (total electron and total nuclear spin angular momenta are zero; see Sec. IV A 4),

$$
\left|\Psi_{T}\right\rangle=\left|X^{1} \Sigma_{g}^{+}(v, J), S_{M}=S_{1}+S_{2}=O, I_{M}=I_{1}+I_{2}=0\right\rangle,
$$

where $S$ is the electron, and $I$ is the nuclear, spin angular momentum. Subscripts $M, 1$, and 2 refer to the molecule and the two atoms, respectively. Dissociated atoms are in the $6^{1} S_{0}$ ground states, for which $S_{1}=S_{2}=0$, and the total angular momentum $F$ is the nuclear spin angular momentum $I$.

The angular momenta can be recoupled so that the spin part of the molecular quantum state is expressed in terms of separated atom basis states $\left|F, M_{F}\right\rangle$,

$$
\begin{aligned}
|\Psi\rangle= & \sum_{M_{F_{1}}, M_{F_{2}}}(-1)^{F_{1}-F_{2}} \\
& \times\left(\begin{array}{ccc}
F_{1} & F_{2} & 0 \\
M_{F_{1}} & M_{F_{2}} & 0
\end{array}\right)\left|F_{1}, M_{F_{1}}\right\rangle_{1}\left|F_{2}, M_{F_{2}}\right\rangle_{2} .
\end{aligned}
$$

The 3-j symbol couples $F_{1}$ and $F_{2}$ and their $z$ components to give zero for each sum. For ${ }^{199} \mathrm{Hg}$ we have $F_{1}=F_{2}=\frac{1}{2}$, and Eq. (2) becomes

$$
|\Psi\rangle=\frac{1}{\sqrt{2}}\left\{\left|\frac{1}{2},+\frac{1}{2}\right\rangle_{1}\left|\frac{1}{2},-\frac{1}{2}\right\rangle_{2}-\left|\frac{1}{2},-\frac{1}{2}\right\rangle_{1}\left|\frac{1}{2},+\frac{1}{2}\right\rangle_{2}\right\} .
$$

In terms of two-component eigenspinors, this can be written as

$$
|\Psi\rangle=\frac{1}{\sqrt{2}}\left\{\left(\begin{array}{l}
1 \\
0
\end{array}\right)_{1}\left(\begin{array}{l}
0 \\
1
\end{array}\right)_{2}-\left(\begin{array}{l}
0 \\
1
\end{array}\right)_{1}\left(\begin{array}{l}
1 \\
0
\end{array}\right)_{2}\right\} .
$$

As expected, this is identical to the state of the two spin- $\frac{1}{2}$ particles in Bohm's classic version of the ERP Gedankenexperiment [4]. Thus, the spatially separated two-atom system is in an "entangled" state suitable for testing Bell inequalities.

To evaluate the component of angular momentum of one of the atoms in a direction at an angle $\theta$ to the $Z$ axis, the rotation matrix for angular momentum $\frac{1}{2}$ that rotates the state through angle $\theta$ to the $Z$ axis is required,

$$
d^{1 / 2}(\theta)=\left\{\begin{array}{cc}
\cos \frac{\theta}{2} & \sin \frac{\theta}{2} \\
-\sin \frac{\theta}{2} & \cos \frac{\theta}{2}
\end{array}\right\},
$$

together with the projection operator for that component of angular momentum onto the $Z$ axis. The projection operator for $M_{F}=+\frac{1}{2}$ is

$$
P_{+}=\left\{\begin{array}{ll}
1 & 0 \\
0 & 0
\end{array}\right\}
$$

Thus, the probability that $M_{F}=+\frac{1}{2}$ will be observed in the direction $\theta$ is given by the expectation value of the matrix operator,

$$
\mathscr{C}_{+}(\theta)=d^{1 / 2}(-\theta) P_{+} d^{1 / 2}(\theta) .
$$

We define $R_{i+}\left(\theta_{i}\right)$ to be the rate of detection of atoms (singles rate) with $M_{F}=+\frac{1}{2}$ in the direction $\theta_{i}$ at detector $i$, where $i=1$ or 2 ; similarly, $R_{i-}\left(\theta_{i}\right)$ is the singles rate for $M_{F}=-\frac{1}{2}$. We define $R_{++}\left(\theta_{1}, \theta_{2}\right)$ to be the coincidence rate for simultaneous detection of an atom at detector 1 with $M_{F}=+\frac{1}{2}$ in the direction $\theta_{1}$ and of an atom at detector 2 with $M_{F}=+\frac{1}{2}$ in the direction $\theta_{2}$. Definitions are analogous for $R_{-+}\left(\theta_{1}, \theta_{2}\right), R_{+-}\left(\theta_{1}, \theta_{2}\right)$, and $R_{--}\left(\theta_{1}, \theta_{2}\right)$. Assuming that the total number of dimers dissociating per unit time is $N$, then the rates for detection of $\mathrm{Hg}$ atoms with $M_{F}=+\frac{1}{2}$ at the two detectors are 


$$
\begin{gathered}
R_{++}\left(\theta_{1}, \theta_{2}\right)=\eta_{1} \eta_{2} f g N\left\langle\Psi\left|\mathscr{C}_{+}\left(\theta_{1}\right) \mathscr{M} \mathscr{b}_{+}\left(\theta_{2}\right)\right| \Psi\right\rangle \\
R_{1+}\left(\theta_{1}\right)=\eta_{1} f N\left\langle\Psi\left|\mathscr{C}_{+}\left(\theta_{1}\right)\right| \Psi\right\rangle \\
R_{2+}\left(\theta_{2}\right)=\eta_{2} f N\left\langle\Psi\left|\mathscr{C}_{+}\left(\theta_{2}\right)\right| \Psi\right\rangle
\end{gathered}
$$

where $\eta_{1}$ and $\eta_{2}$ are the efficiencies for detection of $\mathrm{Hg}$ atoms arriving at the corresponding detectors. The two detectors are symmetric; they have identical geometries and $f$ is the detector acceptance solid angle; $g$ is the conditional probability that if one of the atoms from a dissociated dimer enters the aperture of one detector, then the other atom from that dimer enters the aperture of the other detector.

From Eqs. (8)-(10) and analogous equations for $R_{-+}$, $R_{--}$, etc., together with $|\Psi\rangle$ from Eq. (4), we find the quantum-mechanical predictions,

$$
\begin{gathered}
R_{++}\left(\theta_{1}, \theta_{2}\right)=R_{--}\left(\theta_{1}, \theta_{2}\right)=\eta^{2} f g N \frac{1}{4}\left[1-\cos \left(\theta_{1}-\theta_{2}\right)\right], \\
R_{+-}\left(\theta_{1}, \theta_{2}\right)=R_{-+}\left(\theta_{1}, \theta_{2}\right)=\eta^{2} f g N \frac{1}{4}\left[1+\cos \left(\theta_{1}-\theta_{2}\right)\right], \\
R_{1+}\left(\theta_{1}\right)=R_{2+}\left(\theta_{2}\right)=R_{1-}\left(\theta_{1}\right)=R_{2-}\left(\theta_{1}\right)=\frac{\eta f N}{2}
\end{gathered}
$$

where, for simplicity, we have taken $\eta_{1}=\eta_{2}=\eta$.

The strong Bell-Clauser-Horne $(\mathrm{BCH})$ inequality requires no auxiliary assumptions and is formulated in terms of the ratio of coincidence rates to singles rates $[4,14]$. Since it requires very efficient detectors, it has never been tested. In the present context, the $\mathrm{BCH}$ inequality is

$$
S\left(a, b, a^{\prime}, b^{\prime}\right)=\frac{R_{++}(a, b)-R_{++}\left(a, b^{\prime}\right)+R_{++}\left(a^{\prime}, b\right)+R_{++}\left(a^{\prime}, b^{\prime}\right)}{R_{1+}\left(a^{\prime}\right)+R_{2+}(b)} \leqslant 1,
$$

where $a, a^{\prime}$ are two values of angle $\theta_{1}$, and $b, b^{\prime}$ are two values of $\theta_{2}$. Similar $\mathrm{BCH}$ inequalities can be written for other combinations of components of $M_{F}$. The QM predictions, Eqs. (11) and (13), give a maximum violation of this $\mathrm{BCH}$ inequality for $a, b, a^{\prime}$, and $b^{\prime}$ equal to $135^{\circ}, 0^{\circ}$, $225^{\circ}$, and $90^{\circ}$, respectively,

$$
S_{\mathrm{QM}}\left(135^{\circ}, 0^{\circ}, 225^{\circ}, 90^{\circ}\right)=1.207 \eta g
$$

To observe a violation of the inequality equation (14), the right-hand side of Eq. (15) must be greater than 1; hence the product of the detector efficiencies $\eta$ and the conditional probability $g$ must satisfy

$$
\eta g \geqslant 0.829
$$

Experimental tests of strong Bell inequalities have not previously been able to satisfy stringent requirements of this type. However, we will find that it can easily be satisfied in this experiment ( $\eta>0.95$ and $g \approx 0.97$ ).

It should be emphasized that Eqs. (11) and (12) depend only on the difference between the two angles; this only occurs for parallel detection planes as depicted in Fig. 1. If the two detection planes are not parallel, the quantummechanical prediction depends on each angle separately, not just their difference (see Appendix). In this latter case the maximum violation of the Bell inequalities occurs for a different set of angles $a, b, a^{\prime}$, and $b^{\prime}$, and the maximum value of the violation is not as large as that having parallel planes.

\section{EXPERIMENTAL DETAILS}

\section{A. Mercury dimers}

An essential part of the experiment lies in the preparation of two ${ }^{199} \mathrm{Hg}$ atoms that are in an entangled state with total nuclear spin $I=0$. This can be achieved through spectroscopically selective dissociation of the appropriate mercury dimers.

\section{1. $\mathrm{Hg}_{2}$ spectroscopic data}

Mercury with natural isotopic abundance contains seven isotopes; the resulting dimers then consist of 28 isotopomers with 15 distinct total mass numbers. There are three isotopomers with mass 398; their percentages of the mercury dimers in a beam of natural $\mathrm{Hg}$ are ${ }^{199} \mathrm{Hg}_{2}, 2.84 \% ;{ }^{198} \mathrm{Hg}$ ${ }^{200} \mathrm{Hg}, 4.67 \%$; and ${ }^{196} \mathrm{Hg}{ }^{202} \mathrm{Hg}, 0.09 \%$. The ${ }^{199} \mathrm{Hg}_{2}$ fraction defines the parameter $\alpha_{1}=0.028$, which is used to estimate the expected coincidence rates (Sec. IV D).

Measured spectroscopic parameters for $\mathrm{Hg}_{2}$ of natural isotopic abundance are given in Table I $[18,19]$. The rota-

TABLE I. Spectroscopic parameters of $\mathrm{Hg}_{2}$ with natural isotopic abundance.

\begin{tabular}{lcc}
\hline \hline & $X 0_{g}^{+1} \Sigma_{g}^{+}$ & $D 1_{u}{ }^{3} \Sigma_{u}^{+}$ \\
\hline & & \\
$r_{e}(\AA)$ & $3.63 \pm 0.04^{\mathrm{a}}$ & $2.5 \pm 0.1^{\mathrm{a}}$ \\
$D_{e}\left(\mathrm{~cm}^{-1}\right)$ & $350 \pm 20^{\mathrm{b}}$ & $8260 \pm 200^{\mathrm{b}}$ \\
$T_{e}\left(\mathrm{~cm}^{-1}\right)$ & 0.0 & $31500 \pm 200^{\mathrm{c}}$ \\
$\omega_{e}\left(\mathrm{~cm}^{-1}\right)$ & $18.5 \pm 0.5^{\mathrm{a}}$ & $133 \pm 1^{\mathrm{b}}$ \\
$\omega_{e} x_{e}\left(\mathrm{~cm}^{-1}\right)$ & $0.27^{\mathrm{a}}$ & $0.52 \pm 0.02^{\mathrm{b}}$ \\
$B_{e}\left(\mathrm{~cm}^{-1}\right)$ & $0.0127 \pm 0.0003^{\mathrm{a}}$ & $0.027 \pm .002^{\mathrm{d}}$ \\
$B_{\sim 60}\left(\mathrm{~cm}^{-1}\right)$ & & $0.0177 \pm 0.003^{\mathrm{a}}$ \\
$\alpha_{e}\left(\mathrm{~cm}^{-1}\right)$ & & $(1.5 \pm 0.3) \times 10^{-4} \mathrm{e}$ \\
\hline \hline
\end{tabular}

${ }^{\mathrm{a}}$ Reference [18].

${ }^{\mathrm{b}}$ Reference [19].

${ }^{\mathrm{c} C a l c u l a t e d}$ from the separated atom $\left({ }^{3} P_{1}+{ }^{1} S_{0}\right)$ limit and the values of $D_{e}$.

${ }^{\mathrm{d} C a l c u l a t e d}$ from $r_{e}\left(1_{u}\right)$.

${ }^{\mathrm{e}}$ Calculated from $B_{60}=B_{e}-60.5 \alpha_{e}$. 
tional constant $B_{e}$ for the $D^{3} \Sigma_{u}^{+}\left(1_{u}\right)$ state was calculated using van Zee's value for $r_{e}$ in the relationship $B_{e}=\hbar / 2 \pi c m r_{e}^{2}$, where $m$ is the mass of ${ }^{199} \mathrm{Hg}$. The constant $\alpha_{e}$ is determined from $B_{e}$ and the measured value for the rotational constant $B_{60}$ using $B_{\nu}=B_{e}-(\nu+0.5) \alpha_{e} . T_{e}$ was calculated by taking the energy separation in the separated atom limit between a ${ }^{3} P_{1}+{ }^{1} S_{0}$ and a ${ }^{1} S_{0}+{ }^{1} S_{0}$ configuration, and then correcting for the binding energies $D_{e}$ in the bound $X^{1} \Sigma_{g}^{+}$and excited $D^{3} \Sigma_{u}^{+}$states, respectively.

For excitation in the 266-nm region, the fluorescence excitation spectrum of the $D^{3} \Sigma_{u}^{+} \leftarrow X^{1} \Sigma_{g}^{+}$transition of $\mathrm{Hg}_{2}$ shows a Franck-Condon vibrational progression with a peak in the vicinity of $\nu^{\prime}=58[18,19]$. Each vibrational band consists of a group of subbands separated by $\approx 5 \mathrm{~cm}^{-1}$. Each of these subbands corresponds to one of the 15 distinct dimer mass numbers; only 11 have been observed because of the small natural abundance of ${ }^{196} \mathrm{Hg}$ and ${ }^{204} \mathrm{Hg}$. Each subband in turn consists of separate groups of rotational transitions corresponding to each different isotopomer of the same total mass. It should be noted that for the band heads and assignments of the vibrational levels in the $D^{3} \Sigma_{u}^{+} \leftarrow X^{1} \Sigma_{g}^{+}$transition of $\mathrm{Hg}_{2}$, the two sets of data in the literature do not agree $[18,19]$. Resolution of this discrepancy would be an integral part of this experiment.

\section{Mercury dimer production}

Mercury dimers will be produced in a pulsed supersonic jet source. The dimer beam is on the $X$ axis (cf. Fig. 1) and is collimated to a $600-\mu \mathrm{m}$ diameter. Supersonic jet sources have been run with pure $\mathrm{Hg}$ [20] and various carrier gases $[18,19,21,22]$. Using $\mathrm{Xe}$ as a carrier gas minimizes the dimer velocity, which in turn maximizes the parameter $g$ of Eq. (16) (cf. Sec. IV A 6). Dimer concentration, as well as other beam characteristics, depend on the $\mathrm{Hg}$ vapor pressure, the ratio of carrier gas to $\mathrm{Hg}$ pressure, the oven temperature, and the nozzle shape [22]. Under the same conditions of temperature and pressure, dimer concentration increases significantly when the higher mass Ar rather than $\mathrm{He}$ is the carrier gas [19]. Xe will also be very effective in this regard [23,24].

Operating conditions ultimately have to be optimized experimentally, but good estimates of the operating parameters for the pulsed $\mathrm{Hg}_{2}$ supersonic beam source can be obtained by following prescriptions given in the review article by Miller [25]. Based on the analysis provided by Miller and the data from Table I, we estimate that $5-10 \%$ of the beam will be dimers $\left(\alpha_{2}=0.05\right)$, which is also in good agreement with the work of $\mathrm{Ng}$ [23].

Source temperatures of $\approx 630 \mathrm{~K}$ should be especially favorable for the production of $\mathrm{Hg}_{2}$ dimers in a supersonic beam. In general, pulsed supersonic nozzle sources have been limited to temperatures below $475 \mathrm{~K}$ because of the elastomers used to make a vacuum-tight nozzle seal [26]. However, a source operating at temperatures to $740 \mathrm{~K}$ has been constructed by using KAPTON to seal the nozzle [27]. It is, of course, not essential to have an absolutely vacuumtight seal. The pulse duration will be $\approx 100 \mathrm{msec}$ [28], and the corresponding length of the pulse of atoms for $V_{0}=412$ $\mathrm{m} / \mathrm{sec}$ is $\approx 4 \mathrm{~cm}$.

\section{Mercury dimer rotational populations}

Rotational temperatures in a supersonic beam are difficult to predict. However, van Zee, Blankespoor, and Zwier [18] measured a rotational temperature of $3.5 \mathrm{~K}$ for $\mathrm{Hg}_{2}$ dimers in a $\mathrm{Hg}$ expansion with $\mathrm{Ar}$ as the carrier gas at a source temperature of $\approx 400 \mathrm{~K}$. For present estimates we will also take a rotational temperature of $3.5 \mathrm{~K}$. Assuming a Boltzmann distribution, the fractional population $\eta_{J}$ of the rotational level $J$ is

$$
\begin{aligned}
& \eta_{J}=\frac{3(2 J+1)}{Z} \exp \left(-\frac{h c B_{e} J(J+1)}{k T}\right) \text { for odd } J, \\
& \eta_{J}=\frac{(2 J+1)}{Z} \exp \left(-\frac{h c B_{e} J(J+1)}{k T}\right) \quad \text { for even } J,
\end{aligned}
$$

where the rotational constant $B_{e}$ is given in Table $\mathrm{I}$ and $Z$ is the partition function,

$$
\begin{aligned}
Z= & \sum_{J=0,2,4, \ldots}^{\infty}(2 J+1) \exp \left(-\frac{h c B_{e} J(J+1)}{k T}\right) \\
& +3 \sum_{J=1,3,5, \ldots}^{\infty}(2 J+1) \exp \left(-\frac{h c B_{e} J(J+1)}{k T}\right) .
\end{aligned}
$$

The factor-of- 3 difference in the population for odd and even $J$ originates from the triplet and singlet characters of the corresponding state (cf. Sec. IV A 4). Calculations of the rotational state populations show peaks at $J=10$ and 11 , with peak values of $\approx 3 \%\left(\alpha_{3}=0.03\right)$ and $\approx 9 \%$ for the even and odd $J$ values, respectively.

\section{Spin singlet dimer state}

The ${ }^{199} \mathrm{Hg}$ atom is a fermion with nuclear spin $\frac{1}{2}$. Hence the ${ }^{199} \mathrm{Hg}_{2}$ ground-state dimers must have an antisymmetric total wave function with respect to the exchange of the two nuclei in order to conform to the Pauli principle. The overall symmetry of the wave function is given by the symmetries of the electronic, vibrational, rotational, and nuclear spin wave functions, respectively. The two spin- $\frac{1}{2}$ nuclei of ${ }^{199} \mathrm{Hg}_{2}$ form either a nuclear spin singlet (antisymmetric, total nuclear spin $I=0$ ) or spin triplet (symmetric, $I=1$ ). The vibrational part is always symmetric since it only depends on the relative nuclear coordinates. If we designate the total angular momentum apart from spin by $N$, then rotational wave functions with even $N$ are symmetric and those with odd $N$ are antisymmetric.

The electronic ground state of ${ }^{199} \mathrm{Hg}_{2}$ is the symmetric ${ }^{1} \Sigma_{g}^{+}$state. Its total electron spin angular momentum is zero; therefore, its total angular momentum $J$ (including spin) is $J=N$. Since the electronic wave function is symmetric, to obtain an overall antisymmetric wave function, the combination of rotational and nuclear spin states must be antisymmetric. Specifically, in the ground electronic state, nuclear spin singlet states are associated with even- $J$, and triplet states with odd- $J$, rotational levels [29].

The electronic excited state is the antisymmetric ${ }^{3} \Sigma_{u}^{+}\left(1_{u}\right)$ state. It is a triplet state, and therefore its total angular momentum $J$ is given by $J=N, N \pm 1$. Since the electronic wave function is antisymmetric, the combination 


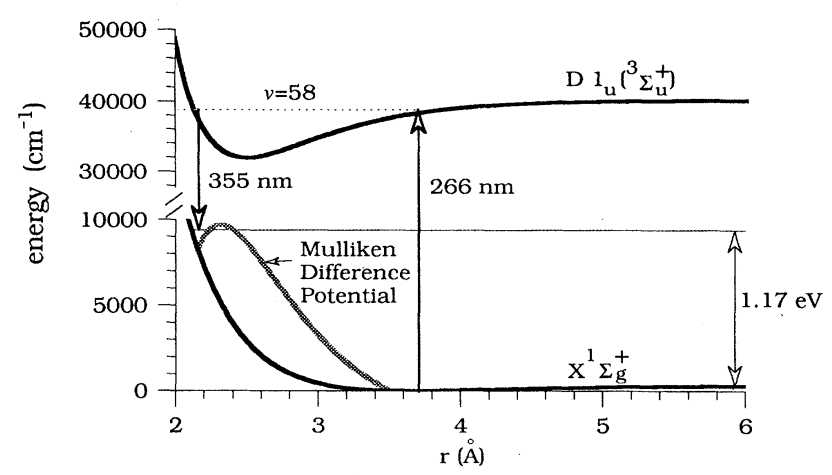

FIG. 2. Energy levels of a mercury dimer and the relevant Raman transitions leading to dissociation. Note the shift in scale on the ordinate. The "Mulliken difference potential" for determining the classical transition points is also shown.

of rotational and nuclear spin states must be symmetric. Specifically, in this state, the nuclear spin singlet states are associated with the odd- $N$ levels, and the nuclear triplet states with the even- $N$ levels [29]. Since the selection rule for the rotational quantum number is that $N$ in the ground state can only differ from $N$ in the excited state by \pm 1 , and since transitions between antisymmetric and symmetric rotational levels are strongly forbidden, it is the change in the symmetry behavior between the rotational levels in the two electronic states that allows transitions between these electronic states of the ${ }^{199} \mathrm{Hg}_{2}$ dimer.

\section{5. $\mathrm{Hg}_{2}$ dissociation}

Dissociation is accomplished via a stimulated Raman transition (see Fig. 2). First a laser system at $266 \mathrm{~nm}$ drives the $P(10)$ transition, in the $\nu$ 58-0 band of the $D^{3} \Sigma_{u}^{+} \leftarrow X^{1} \Sigma_{g}^{+}$system of the isotopomer ${ }^{199} \mathrm{Hg}_{2}$. A second laser at $355 \mathrm{~nm}$ completes a stimulated Raman transition to a continuum level of the ground state and leaves the dissociated atoms with a center-of-mass (c.m.) kinetic energy of $1.17 \mathrm{eV}$. Thus in the c.m. each atom has speed $v=753 \mathrm{~m} / \mathrm{sec}$, given by

$$
v=\sqrt{\left[h \nu_{1}-h \nu_{2}-D_{e}+B_{e} J(J+1)\right] / m},
$$

where $D_{e} \approx D_{0}$ is the dissociation energy, $J$ is the rotational state of the ground state to be dissociated, $B_{e}$ is the rotational constant, $m$ is the mass of a ${ }^{199} \mathrm{Hg}$ atom, and $\nu_{1}$ and $\nu_{2}$ are the frequencies of the 266-nm and the 355-nm photons, respectively.

The radiation at $266 \mathrm{~nm}$ can be produced by third harmonic generation (THG) of the 798-nm output of a pulsed (120-nsec) Alexandrite laser operating on a single longitudinal mode. Nonlinear mixing is accomplished in two steps: first, the second harmonic is generated in a lithium triborate (LBO) crystal that is phase matched for type $\mathrm{I}$ at $31.9^{\circ}$; second, this doubled frequency radiation is sum frequency mixed with the fundamental radiation in a $\beta$-barium borate (BBO) crystal that is phase matched for type I at $44.4^{\circ}$. The dissociation radiation at $\approx 350 \mathrm{~nm}$ can be produced by an excimer-pumped dye laser operating with the dyes BMQ or RDC360.
Since on-resonant transitions will be used in both steps of the Raman process, dissociation rates can be estimated by using resonant excitation and stimulated emission pumping rates together with a rate equation approach (as will be used in Sec. IV B 2). Unfortunately, reliable overall transition moments (equivalently electronic transition moments and Franck-Condon factors) for the transition between the ground state and $\nu=58$ of the $D^{3} \Sigma_{u}^{+}$excited state are not available for such calculations. But these rates are not critical; it is only necessary to demonstrate, that there is sufficient laser power to dissociate an appreciable fraction $\left(\alpha_{4}>0.5\right)$ of the dimers.

For calculating the excitation rate we require the spontaneous emission probabilities from $D^{3} \Sigma_{u}^{+}$. These have been reported as a function of internuclear separation; they vary from $A=7 \times 10^{5} \mathrm{sec}^{-1}$ at the bottom of the $D^{3} \Sigma_{u}^{+}$well to a peak $A=7.6 \times 10^{6} \mathrm{sec}^{-1}$, which corresponds to the $\nu \approx 22$ vibrational state of $D^{3} \Sigma_{u}^{+}[30,31]$. For present estimates we will assume a value at the low end, $A \approx 10^{6} \mathrm{sec}^{-1}$. Based on an analysis similar to that of Sec. IV B 2, the excitation rate is then $R_{a b} \approx 4 \times 10^{13} \mathscr{J}_{a} \mathrm{sec}^{-1}$, where $\mathscr{J}_{a}$ is the $266 \mathrm{~nm}$ spectral intensity ( $\mathrm{W} \mathrm{cm} \mathrm{cm}^{-2} \mathrm{~Hz}^{-1}$ ). If we assume a pulse with an energy $E(\mathrm{~mJ})$, a laser beam diameter of $1.0 \mathrm{~mm}$, and a transform limited full width at half maximum (FWHM) time-bandwidth product of $440 \mathrm{nsec} \mathrm{MHz}$ (for example, temporal and spectral widths of $100 \mathrm{nsec}$ and $4.4 \mathrm{MHz}$, respectively), then $R_{a b} \approx 1 \times 10^{11} E \mathrm{sec}^{-1}$. thus a pulse energy of only $0.1 \mathrm{~mJ}$ gives $R_{a b} \approx 10^{10} \mathrm{sec}^{-1}$; we will have orders of magnitude more energy available. It should also be noted that the fluorescence intensity from $\mathrm{Hg}_{2}$ has a peak at excitation wavelengths near $266 \mathrm{~nm}[18,19]$.

Similarly, for the stimulated emission at $355 \mathrm{~nm}$, large transition probabilities will be obtained for relatively small pulse energies. Since the same two electronic states are involved, the electronic transition moment is as large as before. The critical issue is the Franck-Condon factors and their interference structure for these bound-free transitions [32,33]. Although these have not yet been calculated, the results in Fig. 2 indicate that they will have maximal values. In particular, the "Mulliken difference potential" intersects our final state, i.e., the unbound energy level of $X^{1} \Sigma_{g}^{+}$at 9400 $\mathrm{cm}^{-1}$. These intersection points are classical transition points, where both the nuclear momenta and the nuclear positions are conserved during the transition. Thus, they are points of stationary phase, and their contribution to the integrals for the Franck-Condon factors will be appreciable.

The three different naturally occurring isotopomers of dimer mass 398 (i.e., the possible combinations ${ }^{198} \mathrm{Hg}^{200} \mathrm{Hg},{ }^{196} \mathrm{Hg}^{202} \mathrm{Hg}$, and ${ }^{199} \mathrm{Hg}_{2}$ ) are also spectroscopically differentiated in the corresponding subband of the 58-0 band. In the excitation step at $266 \mathrm{~nm}$ the calculation of the shifts in the transition frequencies between these isotopomers is straightforward [34]. For $\nu=58$, the shifts are dominated by the changes in $\omega_{e}^{\prime}$ and $\omega_{e}^{\prime} x_{e}^{\prime}$ in the excited state. Since the change in $B_{e}$ is negligible, the relative frequencies for each isotopomer are the same as for the ${ }^{199} \mathrm{Hg}_{2}$ dimer. Thus, with respect to the transitions of ${ }^{199} \mathrm{Hg}_{2}$, the transitions of the isotopomers ${ }^{198} \mathrm{Hg}^{200} \mathrm{Hg}$ and ${ }^{196} \mathrm{Hg}^{202} \mathrm{Hg}$ are identical, but are all shifted to higher frequency by 1.6 and $14 \mathrm{GHz}$, respectively. Hence the closest of the other isotopomer transi- 


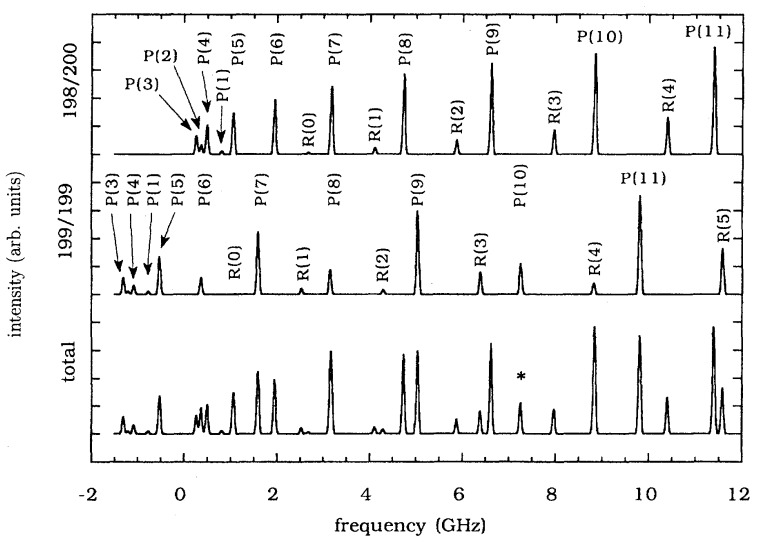

FIG. 3. Simulated rovibrational spectrum for the 58-0 band of the $D^{3} \Sigma_{u}^{+} \leftarrow X^{1} \Sigma_{g}^{+}$transition for the ${ }^{198} \mathrm{Hg}^{200} \mathrm{Hg}$ (top) and the ${ }^{199} \mathrm{Hg}_{2}$ (middle) isotopomers using natural abundance. Because of the large frequency shift of the ${ }^{196} \mathrm{Hg}^{202} \mathrm{Hg}$ transitions, no transitions of this isotopomer are visible in the range of the simulation. As shown in the total spectrum (bottom) the $P(10)$ transition (*) is well resolved. The numbers correspond to the $J$ quantum numbers of the ground state. The simulations show the 3:1 transition intensity alternation due to the spin statistics of the corresponding rotational levels for the ${ }^{199} \mathrm{Hg}_{2}$ dimer (cf. Sec. IV A 4). A rotational temperature of $3.5 \mathrm{~K}$ and a laser linewidth of $60 \mathrm{MHz}$ were assumed.

tions to $P(10)$ of ${ }^{199} \mathrm{Hg}_{2}$ is $P(9)$ of ${ }^{198} \mathrm{Hg}^{200} \mathrm{Hg}$, which is separated by $\approx 600 \mathrm{MHz}$. This is much greater than both the laser linewidth and the natural linewidth. The latter depends on the vibrational state and is given by the spontaneous transition probabilities. Even for the largest of these, $A=7.6 \times 10^{6} \sec ^{-1}[30,31]$, the natural linewidth is only 1.2 $\mathrm{MHz}$; i.e., it is much less than the frequency separation of adjacent transitions. In any event, $\mathrm{Hg}$ atoms from these other isotopomers would be ignored by the detection system due to both frequency mismatch (Sec. IV B 1) and time of flight (Sec. IV A 6).

Figure 3 shows a simulated rovibrational spectrum for the 58-0 band of the $D^{3} \Sigma_{u}^{+} \leftarrow X^{1} \Sigma_{g}^{+}$transition for the two mass 398 isotopomers, ${ }^{199} \mathrm{Hg}_{2}$ and ${ }^{198} \mathrm{Hg}^{200} \mathrm{Hg}$ : natural abundance is assumed. Because of the large frequency shift for the ${ }^{196} \mathrm{Hg}^{202} \mathrm{Hg}$ isotopomer, none of its transitions is within the range of the plot; in addition, on the scale of this plot, their intensity would be vanishingly small because of the low abundance of this isotopomer. Only $R$ and $P$ branches are shown since the splitting constants for the three $J$ values corresponding to each $N$ cannot be determined from existing data $[18,19]$. For the simulations a rotational temperature of $3.5 \mathrm{~K}$ and a laser linewidth of $60 \mathrm{MHz}$ were assumed. The simulations show the $3: 1$ transition intensity alternation due to the spin statistics of the corresponding rotational levels for the ${ }^{199} \mathrm{Hg}_{2}$ dimer (cf. Sec. IV A 4).

In summary, for a test of the Bell inequality, Eq. (14), the dissociating dimers must have total nuclear spin $I=0$, a nuclear spin singlet state. Based on the discussion in Sec. IV A 4, only transitions starting with even $J$ can be used. In particular, because of the angular-momentum selection rules for the excitation $(266 \mathrm{~nm})$ and stimulated emission (355 $\mathrm{nm})$ transitions, the final dissociating level of the $X^{1} \Sigma_{g}^{+}$ ground state must also have even $J$ and hence zero total nuclear spin. Specifically, the final state $J$ differs from the initial state $J$ by $0, \pm 2$. The fact that these transitions are electronic singlet-triplet intercombination lines does not alter this conclusion since $N$ must still change by \pm 1 in each transition [29]. The $P(10)$ transition is particularly favorable, both because the rotational state population has a peak at $J=10$ (as discussed in Sec. IV A 3) and because it is well resolved.

\section{Conditional detection probability $g$}

The conditional detection probability $g$ of Eq. (8) must be as large as possible [see Eq. (16)]. It is determined by the size of the dissociation volume, and the angular distribution of the dissociating dimer fragments, the size and position of the detectors, and the spread in the velocities of the dissociating fragments.

For the dissociation process the $355-\mathrm{nm}$ laser beam has a diameter of $1.5 \mathrm{~mm}$ and is incident along the $Z$ axis (Fig. 1); the 266-nm laser beam has a diameter of $1.0 \mathrm{~mm}$ and lies in the $X-Z$ plane at an angle $\zeta \approx 10^{\circ}$ to the dimer beam. The source volume for the atom pairs is the common intersection of these two laser beams and the supersonic dimer beam. Thus it is a cylinder coaxial with the $X$ axis, with a diameter of $600 \mu \mathrm{m}$ and a length of $1.5 \mathrm{~mm}$. Both lasers have linear polarizations in the $Y$ direction so as to produce a maximum number of dissociated atom pairs in the directions to the two detectors. Specifically, in the c.m. the atom distribution peaks in the direction of the linear polarization of the lasers. For $J=10$ the fraction of dissociated atoms in a small solid angle in this direction is a factor $\kappa=3.8$ greater than if they were isotropically distributed [35].

Momentum conservation requires that, in the c.m. each pair of $\mathrm{Hg}$ atoms must have equal and opposite velocities. Furthermore, in the c.m. all $\mathrm{Hg}$ atoms produced by the dissociation process have essentially the same speed. The spread in their c.m. speeds is determined by the very narrow frequency spread of the dissociating lasers. Their directions are spread over $4 \pi \mathrm{Sr}$ in the c.m. with peaks in the distribution in the direction of the linear polarization of the dissociating lasers [35].

However, for a given direction $\Theta$ in the c.m. the directions $\Psi_{1}, \Psi_{2}$ and the velocities $V_{1}, V_{2}$ in the laboratory frame are determined by vector addition of the dimer velocity $V_{0}$ with the c.m. velocity of the corresponding $\mathrm{Hg}$ atoms (cf. Fig. 4). The detectors and apertures are positioned at these laboratory angles.

With the detector geometry fixed at these angles, it is clear that the smaller the spread in the velocities of the $\mathrm{Hg}_{2}$ dimers the higher the conditional probability. Based on the mean speed $V_{0}=412 \mathrm{~m} / \mathrm{sec}$ and the estimated speed ratio $S=36$ in the supersonic expansion, the spread in dimer velocities is $\Delta V \approx 19 \mathrm{~m} / \mathrm{sec}$, FWHM. For this velocity spread, optimum detector positions, and a usable source size, the conditional probability in Eq. (16) is $g<0.9$.

To obtain larger values of $g$, the Doppler effect in the transition step at $266 \mathrm{~nm}$ will be used to spectroscopically dissociate only dimers within a velocity spread $\Delta V=3$ 


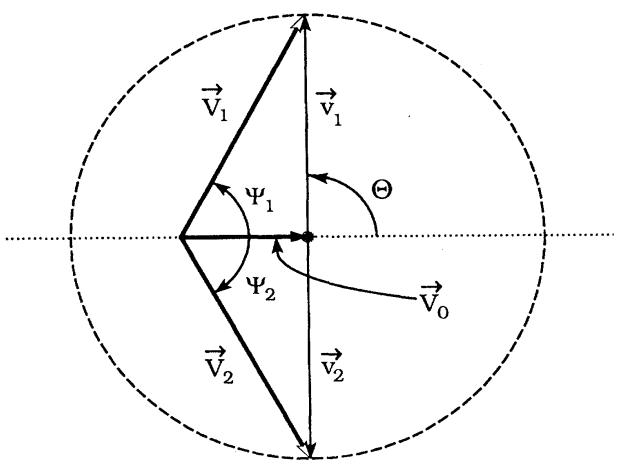

FIG. 4. Velocity transformation diagram. The c.m. velocities of the two mercury atoms are $\vec{v}_{1}$ and $\vec{v}_{2}$; their laboratory velocities are $\vec{V}_{1}$ and $\vec{V}_{2}$. The velocity of the mercury dimer is $\vec{V}_{0}$.

$\mathrm{m} / \mathrm{sec}$. Specifically, if the resonant frequency of a transition is $\omega_{0}$, the laser frequency is $\omega$, and the dimer velocity is $\vec{V}$, then the Doppler effect gives $\omega=\omega_{0}+\vec{k} \cdot \vec{V}$. Here $\vec{k}$ is the wave vector for $266-\mathrm{nm}$ laser radiation and is an angle $\zeta \approx 10^{\circ}$ to the dimer beam. If the laser frequency spread is $\Delta \omega=2 \pi \Delta \nu$ (FWHM), then the corresponding spread in dimer velocities that are resonant with it is $\Delta V \approx \lambda \Delta \nu$. To obtain $\Delta V \leqslant 3 \mathrm{~m} / \mathrm{sec}$ at $266 \mathrm{~nm}$, the laser must have a bandwidth $\Delta \nu \leqslant 11.0 \mathrm{MHz}$. Only a fraction $\alpha_{5} \approx 0.15$ of the dimers can be dissociated because of such a velocity selection. The required narrow linewidth is directly obtained in our proposed laser scheme. The $120-$ nsec pulses produced by the Alexandrite laser have a transform limited bandwidth of 3.7 $\mathrm{MHz}$, resulting in a linewidth of $11 \mathrm{MHz}$ for the third harmonic radiation.

Monte-Carlo simulations were used to evaluate $g$ as a function of the size of the dissociation volume. A brief discussion of the parameters that were used follows:

(i) The dissociation region is cylindrical with a length of $1.5 \mathrm{~mm}$ and radius $r$.

(ii) The density of dissociating dimers is uniform throughout the source volume.

(iii) For simplicity, the velocity distribution of dissociating dimers is assumed to be Gaussian with FWHM $=3 \mathrm{~m} / \mathrm{sec}$ and mean $V_{0}=412 \mathrm{~m} / \mathrm{sec}$.

(iv) For $J=10$, the angular distribution of the dissociating fragments in the c.m. is given by a $12 \cos ^{4} \Theta+10 \cos ^{2} \Theta$ distribution [35]. It should be noted, however, that for the small solid angles relevant to a practical experiment, the differences in the value of the conditional probability $g$ for pure $\cos ^{4} \Theta, \cos ^{2} \Theta$, or uniform distributions are negligible; they only affect the magnitude of the overall count rate. Therefore, uniform fragment distributions were assumed in the Monte Carlo simulations.

(v) The atom speed in the c.m. is $753 \mathrm{~m} / \mathrm{sec}$ (see Sec. IV A 5). It should be noted that both fragments always have identical components of velocity $V_{y}$ toward their respective detectors; of course, the magnitude of $V_{y}$ depends on the c.m. angle $\Theta$.

(vi) The detector apertures are centered on the $X-Y$ plane at an angle corresponding to $\Theta= \pm 90^{\circ}$ in the c.m.; their planes are parallel to the $X-Z$ plane (cf. Fig. 1). The corresponding laboratory angles are $\Psi= \pm 61.3^{\circ}$, and the distance
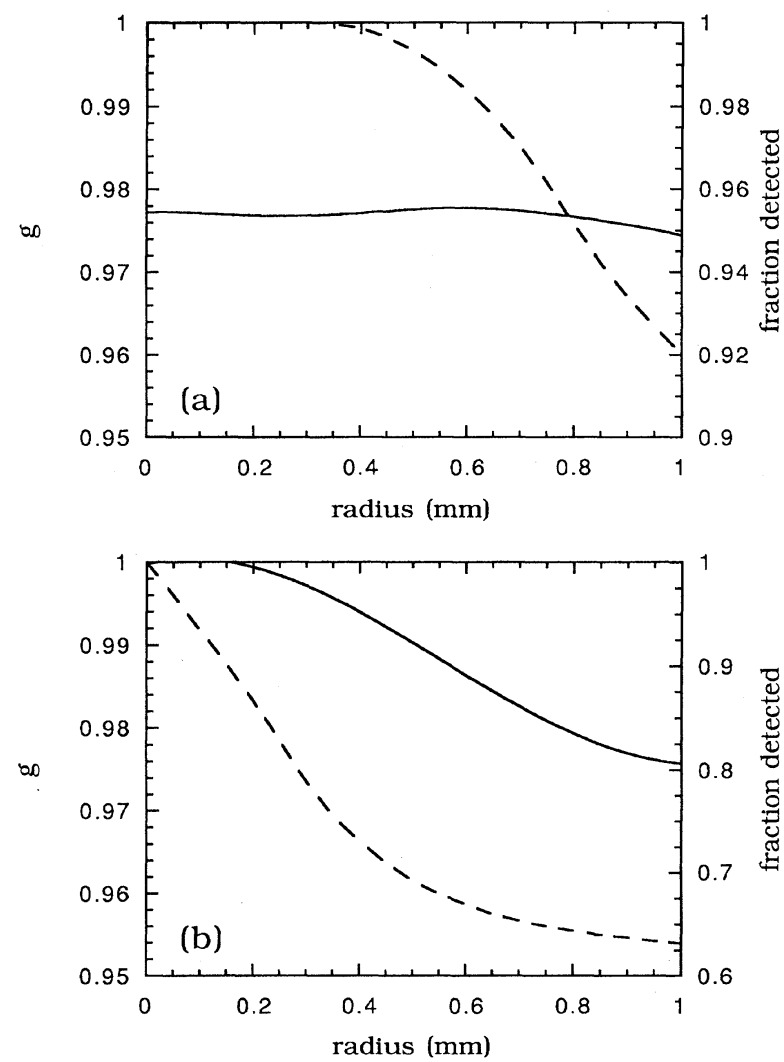

FIG. 5. The solid line is the conditional probability $g$ that if one atom from a dimer enters a detector, the second atom from that dimer will enter the other detector. The dashed line is the fraction of atom pairs that enter both detectors and are within the depth of the detection volume. Both are plotted as a function of the radius of the cylindrical dissociation region, whose length is $1.5 \mathrm{~mm}$, and for two different depths of the detection region: (a) $0.15 \mathrm{~cm}$ and (b) 0.05 $\mathrm{cm}$. In (b), $g$ is enhanced considerably because only atoms within a well-defined arrival time are ionized. In (a), the dip near $r_{s}=0.3$ $\mathrm{mm}$ in the conditional probability $g$ originates from competing mechanisms: the increase in the dissociation volume reduces $g$, and the decrease in the fraction of detected atoms increases $g$.

from the dissociation region to the detection and analyzing regions is $r_{d}=50 \mathrm{~cm}$. The detector apertures in the laboratory frame are circular, with a diameter of $4 \mathrm{~cm}$.

(vii) The detection volume has a depth of $0.15 \mathrm{~cm}$. This is realized by collimating the detection lasers to a crosssectional area $4.0 \times 0.15 \mathrm{~cm}^{2}$ (see Sec. IV B 3). This depth is sufficient to ensure that all ${ }^{199} \mathrm{Hg}$ atom fragments entering the detectors are exposed to the detection lasers; it is also small enough to ensure that a $\mathrm{Hg}$ isotope from any other dissociating isotopomer is not in the detection volume because of its time of flight.

The simulations yield a value of $g=0.976$ for a dissociation (source) region that is $0.6 \mathrm{~mm}$ in diameter and $1.5 \mathrm{~mm}$ long [cf. Fig. 5(a)]. By reducing the fraction of atoms actually detected to about $79 \%, g$ can be increased to 0.997 . This is achieved by simply reducing the detection volume depth to $0.05 \mathrm{~cm}$ so that only atoms in a well-defined time of arrival at the detectors can be detected. This approach is effective in increasing $g$ because it restricts the possible combinations of 


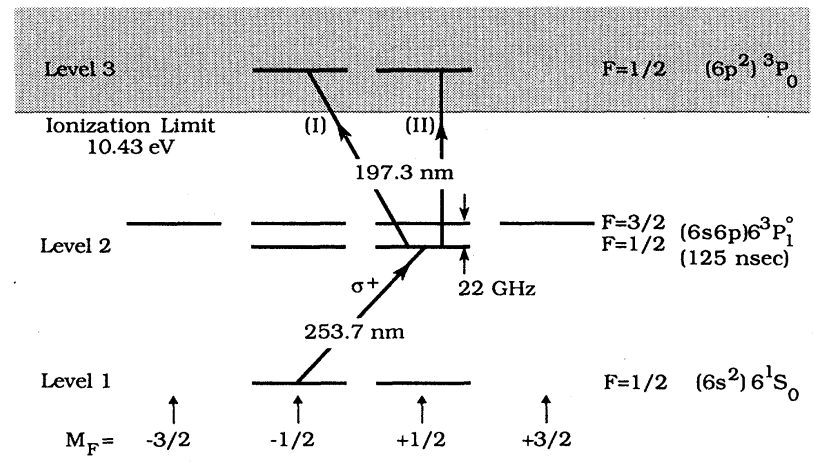

(a)

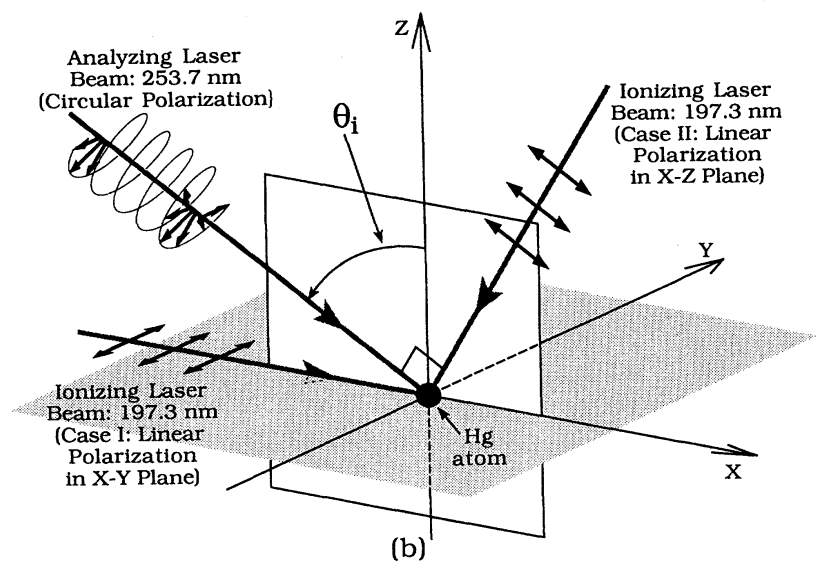

FIG. 6. (a) Relevant energy levels of the $\mathrm{Hg}$ atom and the corresponding transitions for detection. This example shows an angular-momentum analysis using right circularly polarized light. Since $M_{F}$ must increase by 1 , only ground-state atoms with $M_{F}=-\frac{1}{2}$ can be excited to the $6^{3} P_{1}^{o}\left(F=\frac{1}{2}\right)$ state. Cases I and II correspond to different laser alignment and polarization schemes for the transition to the autoionizing state, as shown schematically in (b).

dissociation angles and points of origin of the fragments within the source volume.

\section{B. Mercury atom detection}

As discussed in Sec. III, the highest possible efficiency for detecting both of the $\mathrm{Hg}$ atoms from each dimer is required to test the strong Bell inequalities [cf. Eq. (16)]. High detection efficiency $\eta$ is achieved by using a two-step excitation-ionization process. Immediately following the entrance aperture to each detector (see Fig. 1), the $\mathrm{Hg}$ atoms pass through two laser beams with wavelengths of 253.7 and $197.3 \mathrm{~nm}$. As shown in Fig. 6(a), the first laser is circularly polarized and drives the transition from the $\left(6 s^{2}\right) 6^{1} S_{0}(F=$ $\left.\frac{1}{2}\right)$ ground state (level 1) to the $(6 s 6 p) 6^{3} P_{1}^{o}\left(F=\frac{1}{2}\right)$ state (level 2). The second laser drives the transition from level 2 to the $\left(6 p^{2}\right)^{3} P_{0}$ autoionizing state (level 3); two cases for its polarization and orientation will be considered [cf. Fig. 6(b)]. At the point of ionization the $\mathrm{Hg}^{+}$energy is $0.76 \mathrm{eV}$ (for symmetric dissociation velocities in the laboratory frame) and the photoelectron energy is $0.74 \mathrm{eV}$. Atom detection is via both the resulting ion and the photoelectron.

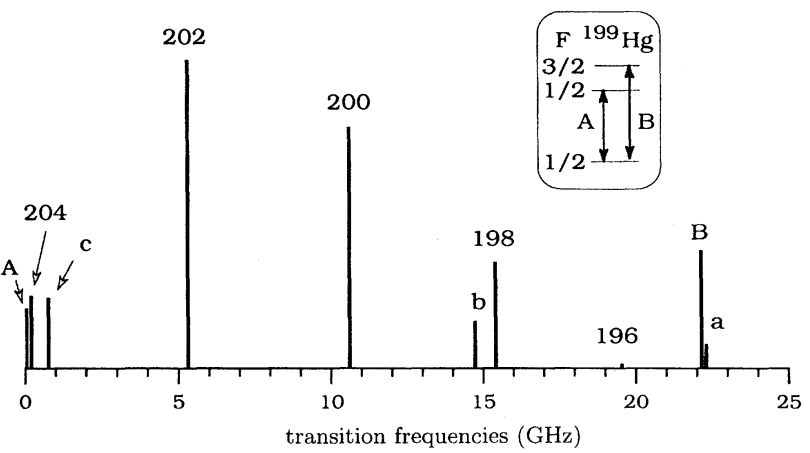

FIG. 7. $6{ }^{1} S_{0} \rightarrow 6^{3} P_{1}^{o}$ transition frequencies for $\mathrm{Hg}$ isotopes relative to the desired ${ }^{199} \mathrm{Hg}$ transition (labeled A). Lowercase letters indicate three of the transitions in ${ }^{201} \mathrm{Hg}$. The isotope ${ }^{204} \mathrm{Hg}$ is suppressed in the dissociation step (cf. Sec. IV A 5).

\section{Atomic excitation rates}

From the measured value of the spontaneous emission probability $A_{2}$ for the $(6 s 6 p) 6^{3} P_{1}^{o}$ state (equivalently its lifetime), the induced absorption probability per unit time $R_{12}$ for the $253.7 \mathrm{~nm}$ excitation transition can be evaluated [36],

$$
R_{12}=\frac{g_{2}}{g_{1}} \frac{\lambda^{3}}{8 \pi h c^{2}} \mathscr{J}_{1}(\nu) A_{2} .
$$

Here $\mathscr{J}_{1}(\nu)$ is the spectral intensity (power per unit area per unit frequency interval $d \nu$ ), and $g_{i}$ is the statistical weight of state $i$. Using $A_{2}=8 \times 10^{6} \mathrm{sec}^{-1}$, [37] we get

$$
R_{12}=7.85 \times 10^{14} \mathscr{J}_{1} \mathrm{sec}^{-1},
$$

where $\mathscr{J}_{1}$ is the $253.7 \mathrm{~nm}$ intensity in units of $\mathrm{W} \mathrm{cm}{ }^{-2} \mathrm{~Hz}^{-1}$. The $6{ }^{1} S_{0} \rightarrow 6^{3} P_{1}^{o}$ transition frequencies for $\mathrm{Hg}$ isotopes, with respect to the relevant transition of ${ }^{199} \mathrm{Hg}$ (labeled A), are shown in Fig. 7 [38].

\section{Atomic ionization rates}

Two cases for polarization and orientation of the ionizing laser are considered. Cases I and II in Figs. 6(a) and (b). For case I the ionizing laser beam propagates on the $X$ axis and is linearly polarized along the $Y$ axis. For these conditions, the ionization transition probability is independent of the angle $\theta$ of the excitation laser beam, and all of the Zeeman sublevels of the $F=\frac{1}{2}$ and $F=\frac{3}{2}$ states of $6^{3} P_{1}^{o}$ can be driven to the autoionizing $\left(6 p^{2}\right)^{3} P_{0}$ state. For case II the ionizing laser beam propagates in the $X-Z$ plane at right angles to the excitation laser beam; it is linearly polarized in the $X-Z$ plane (i.e., parallel to the propagation direction of the excitation laser). In this case the selection rule is $\Delta m_{F}=0$; consequently, atoms in the Zeeman sublevels $m_{F}= \pm \frac{3}{2}$ of $6^{3} P_{1}^{o}$ cannot be excited to the autoionizing level. In practice, case II is more difficult to implement since the ionizing and excitation laser beams must be kept perpendicular as $\theta$ is varied.

Determination of the average transition probability to the autoionizing state $\left(6 p^{2}\right)^{3} P_{0}$ is made by using the measured width, $\Gamma_{3}=9 \mathrm{~cm}^{-1}$, [39] of this state together with a calculated value, $f_{23}=0.362$, [40] for the oscillator strength of the 
transition $(6 s 6 p) 6^{3} P_{1}^{o} \rightarrow\left(6 p^{2}\right)^{3} P_{0}$. Specifically, the cross section is related to the oscillator strength by $[41-43]$,

$$
\int \sigma_{i j}(\nu) d \nu=\frac{\alpha h}{2 m} f_{i j}
$$

where $\alpha$ is the fine-structure constant. A Fano profile is assumed for the autoionizing transition [44],

$$
\sigma_{F}(\varepsilon)=\sigma_{a} \frac{(q+\varepsilon)^{2}}{1+\varepsilon^{2}}+\sigma_{b}
$$

where $q$ is the line parameter and the cross sections $\sigma_{a}$ and $\sigma_{b}$ are due to the continuum interacting with the discrete state and the noninteracting part of the continuum, respectively. The dimensionless parameter $\varepsilon$ is

$$
\varepsilon=\frac{\nu-\nu_{0}}{c \Gamma_{3} / 2}
$$

where $\nu$ is the frequency $(\mathrm{Hz})$ and $c$ is the speed of light $(\mathrm{cm} / \mathrm{sec})$. The integral on the left-hand side of Eq. (23) can be evaluated by integrating over only the contribution of the line itself, $\sigma_{23}=\sigma_{F}(\nu)-\left(\sigma_{a}+\sigma_{b}\right)[45]$ :

$$
\begin{aligned}
\int \sigma_{23}(\nu) d \nu & =\int_{-\infty}^{+\infty}\left[\sigma_{F}(\nu)-\left(\sigma_{a}+\sigma_{b}\right)\right] d \nu \\
& =\frac{c \Gamma_{3}}{2} \sigma_{a} \int_{-\infty}^{+\infty}\left[\frac{(q+\varepsilon)^{2}}{1+\varepsilon^{2}}-1\right] d \varepsilon \\
& =\frac{c \Gamma_{3}}{2} \sigma_{a}\left(q^{2}-1\right) \int_{-\infty}^{+\infty} \frac{d \varepsilon}{1+\varepsilon^{2}} \\
& =\frac{\pi}{2} \Gamma_{3} c \sigma_{a}\left(q^{2}-1\right)=\frac{\pi}{2} \Gamma_{3} c \sigma_{\mathrm{res}}
\end{aligned}
$$

where $\sigma_{\text {res }}=\sigma_{a}\left(q^{2}-1\right)$ is the value of $\sigma_{23}$ at resonance, $\sigma_{23}\left(\nu_{0}\right)$. The maximum value of $\sigma_{23}$ is $\sigma_{\max }=\sigma_{23}(1 / q)=\sigma_{a} q^{2}$. Since the observed line shape is Lorentzian $[39,46], q$ is large and we can assume $\sigma_{\text {max }} \approx \sigma_{\text {res }}$. With this result, Eq. (23) yields

$$
\sigma_{\max } \approx \frac{\alpha h}{\pi m c} \frac{f_{23}}{\Gamma_{3}}=5.636 \times 10^{-13} \frac{f_{23}}{\Gamma_{3}}=2.3 \times 10^{-14} \mathrm{~cm}^{2},
$$

where $\Gamma_{3}$ is in $\mathrm{cm}^{-1}$. This is a very large cross section, but it is consistent with measured cross sections for analogous transitions in $\mathrm{Cd}$ [47]. If we assume that the laser linewidth $\ll \Gamma_{3}$, the cross section will be nearly constant $\left(\sigma_{\max }\right)$ over the linewidth, and the transition probability per unit time $R_{23}$ is then [48]

$$
R_{23}=\frac{1}{\hbar \omega} \sigma_{\max } \int I(\omega) d \omega=\frac{\lambda}{h c} \sigma_{\max } I_{2}=2.25 \times 10^{4} I_{2} \mathrm{sec}^{-1},
$$

where $I_{2}$ is in $\mathrm{W} \mathrm{cm}^{-2}$.

One can now write rate equations for the populations of the three levels in terms of $A_{2}, R_{12}$, and $R_{23}$. Atoms excited
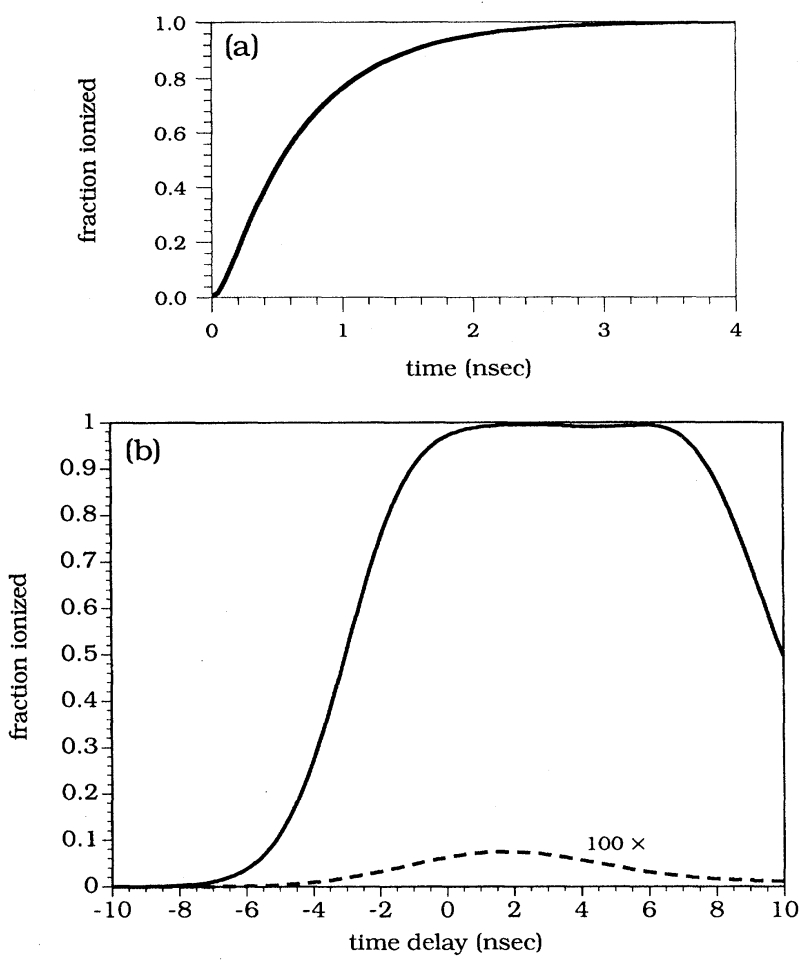

FIG. 8. (a) The fraction of ${ }^{199} \mathrm{Hg}$ atoms ionized as a function of time, assuming the laser intensity is a square pulse turned on at $t=0$. Laser intensities are $\mathscr{J}_{1}=1 \times 10^{-5} \quad \mathrm{~W} \mathrm{~cm}^{-2} \mathrm{~Hz}^{-1}$, $I_{2}=1 \times 10^{+5} \mathrm{~W} \mathrm{~cm}^{-2}$. (b) The fraction of ${ }^{199} \mathrm{Hg}$ atoms ionized as a function of the delay time between the two laser pulses using the numerical solution of the master equations. Positive delay times indicate that the laser pulse connecting levels 1 and 2 is fired first. The dashed curve shows the fraction of ${ }^{199} \mathrm{Hg}$ atoms ionized for case I, assuming a detuning of the first laser by $22 \mathrm{GHz}$ from resonance. This corresponds to the unwanted process that would lead to the loss of spin-state resolution. For clarity, these data were multiplied to 100 . For case II the dashed curve would be zero because of the selection rules. Laser intensities are as in (a).

to level 3 are assumed to ionize (and leave the system). These equations are solved, assuming for simplicity that the time dependence of the intensities of the exciting and ionizing radiation are rectangular and turned on at $t=0$ [49]. Figure 8(a) shows the fraction of atoms ionized as a function of time for $\mathscr{J}_{1}=1 \times 10^{-5} \mathrm{~W} \mathrm{~cm}^{-2} \mathrm{~Hz}^{-1}$ and $I_{2}=1 \times 10^{+5}$ $\mathrm{W} \mathrm{cm}{ }^{-2}$. Essentially all the atoms are ionized within $3 \mathrm{nsec}$.

Since high ionization efficiency is so important, it is necessary to check the ionization probability in the $\left(6 p^{2}\right)^{3} P_{0}$ state. Specifically, the $\left(6 p^{2}\right)^{3} P_{0}$ state can either radiate or autoionize [50]. This could have been included in the rate equations, but we shall see that radiative effects are negligible. Radiative decay is to $(6 s 6 p)^{3} P_{1}^{o}$ and $(6 s 6 p)^{1} P_{1}^{o}$. Decays to all other states are negligible; they are either forbidden $(J=0 \nrightarrow J=0,2)$ or have configurations that would require at least two electron jumps. The ratio of the oscillator strengths for decay to ${ }^{3} P_{1}^{o}$ versus decay to ${ }^{1} P_{1}^{o}$ is 63 [40]. Hence, most radiative decays are to ${ }^{3} P_{1}^{o}$. Neglecting decay to ${ }^{1} P_{1}^{o}$, the radiative lifetime (in terms of the oscillator strength $\left.f_{23}\right)$ is $[51,52]$ 


$$
\tau_{3(\mathrm{rad})}=\frac{g_{3}}{g_{2}} \frac{m}{4 \pi h \alpha} \lambda^{2} \frac{1}{f_{23}}=5.4 \times 10^{-10} \mathrm{sec},
$$

whereas the actual lifetime of the $\left(6 p^{2}\right)^{3} P_{0}$ state (level 3) is

$$
\tau_{3}=\frac{1}{\Gamma_{3}}=3.7 \times 10^{-12} \mathrm{sec}
$$

The fraction of atoms in level 3 that ionize is therefore

$$
\left(1-\frac{\tau_{3}}{\tau_{3(\mathrm{rad})}}\right)=0.993 \equiv 99.3 \%
$$

Thus, less than $0.7 \%$ take the radiative route. But for case I [Figs. 6(a) and (b)] most of these atoms will be kicked right back up to level 3, regardless of the hyperfine level to which they decay, since the linewidth of the transition to $\left(6 p^{2}\right)^{3} P_{0}$ is much greater than the hyperfine splitting in ${ }^{3} P_{1}^{o}$. For case II some of the atoms taking the radiative route will be effectively trapped in the $m_{F}= \pm \frac{3}{2}$ sublevels.

The adiabatic passage pumping scheme for a three-level system should also be examined since it has been shown to yield a $100 \%$ population transfer from the initial to the final level while avoiding any population of the intermediate level $[53,54]$. This pumping scheme is often referred to as "counterintuitive" since the pulse from the intermediate level 2 to the final level 3 is fired before the pulse connecting the initial level 1 with the intermediate level. The important advantage is that there are no losses due to spontaneous emission from the intermediate level. The first laser pulse builds up a coherence between the intermediate and final levels, thereby forming a coherent superposition of states in a dressed-atom picture that enables the efficient pumping scheme.

The requirement of such a pumping scheme is that the Rabi frequencies of the transitions are of the same order as the corresponding widths of the levels. Since the width of the final level is very broad because of the fast autoionization, the coherence between the intermediate and the final levels immediately decays, so that the adiabatic pumping scheme is not effective. Very large Rabi frequencies, i.e., on the order of the width of the autoionizing level, would be required to overcome this decay of coherence. On the other hand, large Rabi frequencies would also involve a mixing between the $F=\frac{3}{2}$ and $\frac{1}{2}$ levels of the $(6 s 6 p)^{3} P_{1}^{o}$ state since the final level is much broader than the $22-\mathrm{GHz}$ separation of these two hyperfine levels. Hence, ground-state atoms with either component of spin would be excited.

In case I, they would all be ionized and the resolution of the spin state would be completely lost; this is, of course, a crucial point of the experiment. In case II, however, as a result of the selection rule $\Delta m_{F}=0$ on the $(6 s 6 p) 6^{3} P_{1}^{o} \rightarrow\left(6 p^{2}\right)^{3} P_{0}$ transition, only one spin state is ionized; the other spin state is excited to an $m_{F}= \pm \frac{3}{2}$ sublevel that is not accessed. Thus in case II the resolution of the spin state would still be maintained.

Nevertheless, the counterintuitive pumping scheme cannot be applied in this experiment, since the anticipated laser intensities are insufficient to achieve the required Rabi frequencies. Even if such intensities were available, practical considerations such as multiphoton ionization of residual gas, photoelectric effects, etc., would make such an approach undesirable.

The degree of ionization was calculated for the previously stated intensities $\left(\mathscr{J}_{1}=1 \times 10^{-5} \mathrm{~W} \mathrm{~cm}^{-2} \mathrm{~Hz}^{-1}\right.$, $I_{2}=1 \times 10^{+5} \mathrm{~W} \mathrm{~cm}^{-2}$ ) as a function of the delay time between the first $\left(\mathscr{J}_{1}\right)$ and second pulse $\left(I_{2}\right)$. The calculations involved the numerical solution of the corresponding master equations assuming Fourier-transform-limited Gaussian pulses of 8 nsec FWHM duration using a Runge-Kutta algorithm with adaptive step size. As shown in Fig. 8(b), a maximum of $99.5 \%$ of the population is transferred to the autoionizing level when the first pulse $(253.7 \mathrm{~nm})$ precedes the second pulse $(197.3 \mathrm{~nm})$ by $2 \mathrm{nsec}$. This pulse sequence is opposite the counterintuitive pumping scheme [i.e., negative delay times in Fig. 8(b)]. The reason for the $0.5 \%$ loss in the population transfer is the possible decay from the intermediate level to the other $m_{F}$ sublevel of the ground state; because of the circular polarization of the first pulse the latter cannot be excited to the intermediate level. Even for case I, transitions through the $m_{F}= \pm \frac{3}{2}$ hyperfine states are highly suppressed $[<0.075 \%$, Fig. 8(b)], and measurement of the nuclear spin state can be achieved. Of course, in case II, all transitions through the $m_{F}= \pm \frac{3}{2}$ hyperfine state are completely suppressed.

It should be noted that several investigators have used photoionization for $\mathrm{Hg}$ detection [55], isotope selection [56], and isomer selection [57]. Two of these tried to take advantage of a broad autoionizing state $[55,57]$, but the oscillator strengths for their transitions to it were so small as to negate any benefits. The proposed scheme (and several variants that increase selectivity) is orders of magnitude more sensitive and will be valuable in these and similar applications.

\section{Detection lasers}

The detection volume is defined by its $0.15-\mathrm{cm}$ depth and the area of the 4-cm-diam aperture. Lasers at wavelengths 253.7 and $197.3 \mathrm{~nm}$ must simultaneously illuminate the entire detection volume. This is achieved by collimating the lasers to a rectangular beam of cross-sectional area 0.15 $\mathrm{cm} \times 4.0 \mathrm{~cm}=0.6 \mathrm{~cm}^{2}$.

For the excitation laser at $253.7 \mathrm{~nm}$, there are several possibilities; as an example, it can be the frequency-tripled output of a Ti:sapphire laser operating at $761.1 \mathrm{~nm}$. Output energies of $\approx 100 \mathrm{~mJ}$ at the fundamental can easily be achieved. Employing the same crystals and tripling scheme as described in Sec. IV A 5, we can obtain greater than $1 \mathrm{~mJ}$ at $253.7 \mathrm{~nm}$ in $\approx 8 \mathrm{nsec}$ pulses with a bandwidth of $\approx 0.01$ $\mathrm{cm}^{-1} \equiv 0.3 \mathrm{GHz}$. This bandwidth completely resolves the hyperfine splitting $(22 \mathrm{GHz})$ of $6^{3} P_{1}^{o}$. The resulting spectral intensity is $>6 \times 10^{-4} \mathrm{~W} \mathrm{~cm}^{-2} \mathrm{~Hz}^{-1}$, which is a factor of 60 larger than the intensity $\mathscr{J}_{1}$ that is required.

The radiation at $197.3 \mathrm{~nm}$ can be obtained by using BBO to frequency sum the fourth harmonic $(266 \mathrm{~nm})$ of a Nd:YAG (neodymium-doped yttrium aluminum garnet) laser with the fundamental of a flash-lamp-pumped Ti:sapphire laser tuned to $764 \mathrm{~nm}$. In fact this Ti:sapphire can be the same as that used for generating the $253.7-\mathrm{nm}$ radiation, as the Ti:sapphire laser can be injection seeded simultaneously with two lasers diodes. Not only does this provide the two wavelengths as single longitudinal mode outputs, but also the 
diode seed lasers make it easy to tune the outputs and lock them to the $\mathrm{Hg}$ transitions.

Previously, Glab and Hessler mixed the fundamental output of a dye laser with its second harmonic in BBO [58]. With pulse energies of $5 \mathrm{~mJ}$ and power densities of 50 $\mathrm{MW} \mathrm{cm}^{-2}$, they observed an efficiency of $\approx 20 \%$ for wavelengths down to $199 \mathrm{~nm}$. The efficiency then dropped sharply, and the shortest wavelength they generated was $197.4 \mathrm{~nm}$. The drop was due to the decrease in the nonlinear optical constants when the phase-matching angle approached $90^{\circ}$. Others obtained wavelengths as short as $195.3 \mathrm{~nm}$ by cooling the BBO crystal [59]. The phase-matching angle of $90^{\circ}$ occurs at a shorter wavelength if the wavelengths for the frequency summing process are more widely separated. By using two different dye lasers, wavelengths down to 188.9 $\mathrm{nm}$ have been obtained [60]. Although efficiencies of only $\approx 3 \%$ were observed, the bandwidths were larger and the power densities were an order of magnitude smaller than in the work by Glab and Hessler.

An appropriate approach is to use a Nd:YAG laser. The latter easily produces $\approx 25 \mathrm{~mJ}$ in an 8 -nsec pulse at the fourth harmonic, $266 \mathrm{~nm}$. The Ti:sapphire laser produces $\approx 100 \mathrm{~mJ}$ in a 20 -nsec pulse at $764 \mathrm{~nm}$; we assume that only $\approx 40 \mathrm{~mJ}$ of this temporally overlaps the $\mathrm{Nd}$ :YAG pulse and will be useful. Now, because of the large difference in wavelengths being summed, the phase-matching conditions are easily satisfied. With indices of refraction based on Kato's results for the coefficients in Sellmeier's equation [61], the phase-matching angle in $\mathrm{BBO}$ for these wavelengths is $68^{\circ}$. It is known that Kato's coefficients give indices in the 200-nm region that are slightly in error and that actual phasematching angles are a little larger [58]. With this phasematching angle, conversion efficiencies of $\approx 20 \%$ (comparable to that of Glab and Hessler) will be observed, and the output power at $197.3 \mathrm{~nm}$ will be $>6 \mathrm{~mJ}$ in an $8-\mathrm{nsec}$ pulse. As before, this will be focused into an area $0.15 \mathrm{~cm} \times 4.0$ $\mathrm{cm}=0.6 \mathrm{~cm}^{2}$. Thus the available intensity is $>1.2 \times 10^{6}$ $\mathrm{W} \mathrm{cm}{ }^{-2}$, a factor of 12 larger than the intensity $I_{2}$ that is required [see Figs. 8(a) and (b)].

Sufficient laser power can clearly be generated to saturate the resonant two-step ionization process. Intensities will, in fact, be reduced to the extent possible while maintaining greater than $99 \%$ ionization. The relatively low power requirement for the second transition is a consequence of the extremely large cross section for excitation of the autoionizing state, and is important since it virtually eliminates multiphoton ionization of residual gas that could produce backgrounds.

\section{Detection efficiency and background}

For 300-eV electrons, the efficiency of cone-input Channeltrons is $>90 \%$ [62] and may reach 98\% [63]. For highmass ions accelerated to $\approx 3 \mathrm{keV}$, the efficiency of cone-iput Channeltrons is $>50 \%$ [64] and may reach $90 \%$ [65]. An ion can also be detected by accelerating it into a metal electrode to produce a burst of electrons. These, in turn, are accelerated into a scintillator to produce a burst of photons that are observed with a photomultiplier tube $[66,67]$. For ion energies $\geqslant 15 \mathrm{keV}$, the ion detection probability is $>90 \%$ [66].

Both the $\mathrm{Hg}^{+}$ion and its photoelectron will be observed, and we will assume that a $\mathrm{Hg}$ atom has been observed if either the electron or the ion detector gives an output pulse. As a result of this or decision, and assuming worst-case values of $50 \%$ and $90 \%$ for the ion and electron detection efficiencies, respectively, the overall efficiency for detection of a $\mathrm{Hg}$ atom is at least $95 \%$ (e.g., at the lower limit, $90 \%$ of the $\mathrm{Hg}$ atoms are detected via the electron, and $50 \%$ of the remaining $10 \%$ are detected via the ion). Actually, overall $\mathrm{Hg}$ atom detection efficiencies $>99 \%$ should be achievable. An important consequence of the or decision is that background or noise counts must be made negligible.

Channeltron dark count rates are $<0.5$ counts/sec. However, detector pulses are only observed in a coincidence time window of $\approx 20 \mathrm{nsec}$ when the detection lasers are fired (10 times/sec). Thus the dark count rate within the coincidence window is $<1 \times 10^{-7}$ counts/sec. This is negligible.

To avoid counts due to residual gas $\mathrm{Hg}$ atoms, the average number in the detection volume $\left(0.15 \times 4 \times \pi \mathrm{cm}^{3}\right)$ must be $\ll 1$; i.e., the $\mathrm{Hg}$ partial pressure must be $\ll 10^{-17}$ Torr. Thus, the detection region walls will be cooled to $77 \mathrm{~K}$ (liquid $\mathrm{N}_{2}$ ), where the vapor pressure of $\mathrm{Hg}$ is estimated to be $\sim 10^{-32}$ Torr [68].

Of course, it is essential to eliminate background signals due to stray electrons and ions. Thus, all high-voltage electrodes must be appropriately treated to suppress field emission, and a cryopumped vacuum of better than $10^{-9}$ Torr must be maintained. The relatively low power densities of the uv detection lasers are also critical to the elimination of background charge-particle sources. Construction materials must be selected and cleaned to eliminate emission of photoelectrons by the uv lasers.

Since there is a one-to-one correspondence between a $\mathrm{Hg}^{+}$ion and its photoelectron, we can compare singles rates and coincidence rates between ion and electron detectors to obtain the absolute overall detection efficiency of both the ion detector and the electron detector [69]. The relations between the count rates and the detector efficiencies are

$$
N_{e}=\eta_{e} N_{a}, \quad N_{i}=\eta_{i} N_{a}, \quad N_{c}=\eta_{i} \eta_{e} N_{a}
$$

where $N_{a}$ is the overall number of atoms entering a detector, and $N_{e}, N_{i}$, and $N_{c}$ are the count rates for electrons, ions, and electron-ion coincidences, respectively. The electron and ion detector efficiencies are $\eta_{i}$ and $\eta_{e}$, respectively. Equations (32) can easily be solved for these efficiencies, yielding

$$
\eta_{i}=\frac{N_{c}}{N_{e}}, \quad \eta_{e}=\frac{N_{c}}{N_{i}} .
$$

Thus, the ion and electron detector efficiencies $\eta_{i}$ and $\eta_{e}$ can be directly measured and continuously monitored throughout all data acquisition. The inherent determination of the detector efficiencies is one of the important and outstanding features of this experiment.

The efficiency measurements for ion and electron detectors (including Channeltrons) will also be of considerable technical interest in their own right. They will provide absolute efficiency measurements that are not encumbered by the usual errors associated with determining the absolute source intensity and geometry. 


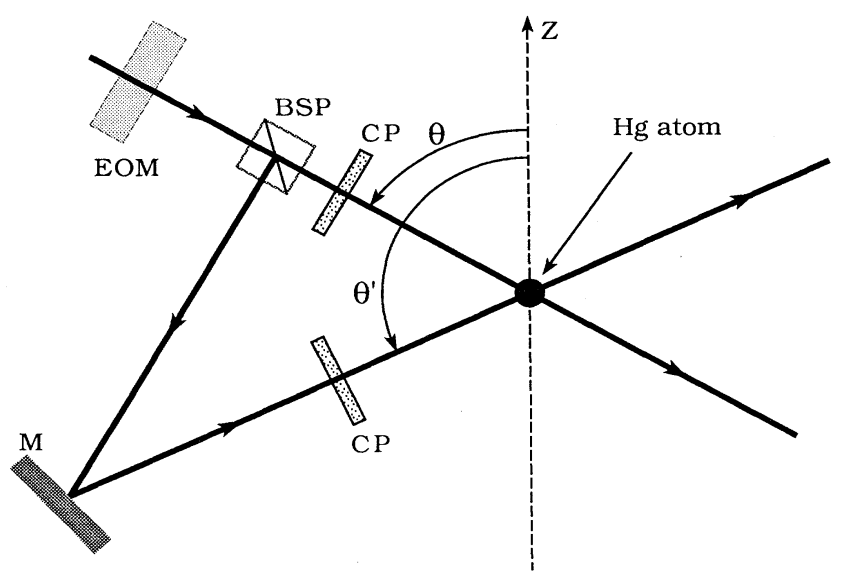

FIG. 9. Schematic of the system for rapid switching of the direction of observation of the nuclear spin components. EOM, electro-optical modulator (switches laser beam between orthogonal linear polarization states on the nanosecond time scale); M, mirror; $\mathrm{CP}$, circular polarizer (transmits one linear polarization state and reflects the orthogonal one).

With the aforementioned or detection scheme the overall detector efficiency $\eta$ in Eqs. (11)-(13) is related to the detection efficiencies $\eta_{i}$ and $\eta_{e}$ by

$$
\eta=1-\left(1-\eta_{e}\right)\left(1-\eta_{i}\right)=\eta_{e}+\eta_{i}-\eta_{e} \eta_{i} .
$$

Thus the absolute detection efficiency for both atoms can be determined in this way. The two detectors do not have to have exactly the same detection efficiency in order to test the strong Bell inequality; Eqs. (11)-(13) can easily be formulated for two detectors with different efficiencies and solid angles. The conditional probability $g$ is measured by observing that the ratio of Eqs. (11) and (13) for a pair of angles $\theta_{1}$ and $\theta_{2}$ yields

$$
\frac{R_{++}\left(\theta_{1}, \theta_{2}\right)}{R_{1+}\left(\theta_{1}\right)}=\frac{1}{2} g \eta\left[1-\cos \left(\theta_{1}-\theta_{2}\right)\right] \text {. }
$$

If $\theta_{1}-\theta_{2}=\pi$, then $R_{++} / R_{1+}=g \eta$. Since $\eta$ has been measured [Eq. (34)], the data for $R_{++}$and $R_{1+}$ give an experimental value for $g$. It should be noted that $g$ can also be determined in a completely independent measurement by using linear rather than circular polarized $253.7-\mathrm{nm}$ radiation; in this case $g \eta$ is the ratio of the coincidence rate to the singles rate, both of which are independent of $\theta_{1}$ and $\theta_{2}$.

\section{Angular-momentum analysis}

As shown in Fig. 1, the analyzing beams at $253.7 \mathrm{~nm}$ lie in planes parallel to the $X-A$ plane and are at angles $\theta_{1}, \theta_{2}$ to the $+Z$ axis. The angles $\theta_{1}, \theta_{2}$ of these laser beams define the directions in which each atom's angular-momentum components is observed.

For a ground-state ${ }^{199} \mathrm{Hg}\left(F=\frac{1}{2}\right)$ atom, the quantum numbers for the two components of angular momentum in a given direction are $M_{F}= \pm \frac{1}{2}$; these correspond to the components of nuclear spin in that direction. If the 253.7-nm laser beam has right circular polarization $\left(\sigma^{+}\right), M_{F}$ must increase by 1 in the transition. Thus, only ground-state atoms for which the projection of the angular momentum (nuclear spin) in the direction of laser beam propagation is $M_{F}=\frac{1}{2}$ can be excited to the $6^{3} P_{1}^{o}\left(F=\frac{1}{2}\right)$ state and subsequently ionized; see Fig. 6(a). Similarly, for left circular polarization, only atoms with $M_{F}=+\frac{1}{2}$ are excited and ionized. In summary, by choosing the direction and circular polarization of the excitation laser, a $\mathrm{Hg}$ atom with either component of nuclear spin in any direction is selectively detected.

Einstein locality can also be rigorously enforced in this analysis scheme. Specifically, as indicated in Fig. 9, an electro-optic modulator (EOM) together with a beamsplitting polarizer can change the propagation direction of the excitation laser beam and hence the component of angular momentum being observed. The EOM can switch the laser beam polarization between orthogonal linear polarization states on the nanosecond time scale. This, as well as the time to digitize the detector output, can be short compared to the time for light to travel between the detector systems. The EOM can be triggered stochastically in a variety of ways.

\section{Signal estimates}

Based on Eq. (11), the maximum coincidence rate $R$ can be written in terms of parameters specified throughout this paper (and summarized in Table II),

TABLE II. Summary of parameters for the signal estimate.

\begin{tabular}{lc}
\hline \hline $\mathscr{R}=$ pulse repetition rate & $10 \mathrm{sec}^{-1}$ \\
$\eta=$ detector efficiencies & 0.95 \\
$g=$ conditional detection probability & 0.97 \\
$n_{0}=$ Hg density in dissociation region & $2.4 \times 10^{11} \mathrm{atoms} \mathrm{cm}^{-3}$ \\
$\alpha_{1}=$ fraction of dimers that are ${ }^{199} \mathrm{Hg}_{2}$ & 0.028 \\
$\alpha_{2}=$ fraction of beam that is dimers & 0.05 \\
$\alpha_{3}=$ fraction of dimers that are in $J=10$ & 0.03 \\
$\alpha_{4}=$ fraction of dimers that are dissociated & 0.5 \\
$\alpha_{5}=$ fraction of dimers in velocity selection window & 0.15 \\
$\kappa=$ increase due to anisotropy of molecular dissociation & 3.8 \\
$r_{s}=$ radius of dissociation region (source) & $0.03 \mathrm{~cm}$ \\
$L=$ length of dissociation region & $0.15 \mathrm{~cm}$ \\
$r_{d}=$ distance from dissociation region to detectors & $50 \mathrm{~cm}^{2}$ \\
$A=$ cross-sectional area of detector & $12.6 \mathrm{~cm}^{2}$ \\
\hline \hline
\end{tabular}




$$
R=\frac{1}{2} \mathscr{B} \eta^{2} g n_{0} \alpha_{1} \alpha_{2} \alpha_{3} \alpha_{4} \alpha_{5} \kappa \pi r_{s}^{2} L \frac{A}{4 \pi r_{d}^{2}} .
$$

We find $R \approx 2$ coincidences/sec. This conservative estimate is already too high; specifically, since $\mathscr{R}=10 \mathrm{sec}^{-1}$, such a large coincidence rate will give an appreciable number of coincidences between atoms from different dimers. An optimal rate with $\mathscr{R}=10 \mathrm{sec}^{-1}$ would be $<1$ coincidence/sec; of course, it is always easy to reduce a signal.

\section{E. Other effects}

\section{Magnetic effects}

The nuclear magnetic moment for ${ }^{199} \mathrm{Hg}$ is $\mu=2.56 \times 10^{-31} \mathrm{~J} \mathrm{G}^{-1}[70]$. It is easy to reduce residual magnetic fields to $B<0.01 \mathrm{G}$. In such a field and for a flight time to the detector of $t=6 \times 10^{-4} \mathrm{sec}$, the magnetic moment precesses through an angle $\theta<0.8^{\circ}$. This is a negligible effect.

\section{Hg atom collisions}

As a least favorable condition, we will consider the mean free path $\lambda_{m}$ of a $\mathrm{Hg}$ atom in $\mathrm{Hg}$ vapor. Using the long-range Lennard-Jones attractive potential, the mean free path is [25],

$$
\lambda_{m}=\left[5.3 n\left(C_{6} / k T\right)^{1 / 3}\right]^{-1}=\frac{9.6 \times 10^{13}}{n} \mathrm{~cm},
$$

where $n$ is the atom density (atoms $\times \mathrm{cm}^{-3}$ ), $T=300 \mathrm{~K}$, and $C_{6} / k$ is the attractive force parameter for $\mathrm{Hg}$. The latter is given by [25]

$$
\frac{C_{6}}{k}=2 \frac{h c D_{e}}{k} r_{e}^{6}=2.3 \times 10^{-42} \mathrm{~K} \mathrm{~cm}^{-1},
$$

where $D_{e}$ and $r_{e}$ are given in Table I. The background pressure is $\approx 10^{-9}$ Torr. Thus, we have $\lambda_{m} \approx 10^{6} \mathrm{~cm} \gg 50 \mathrm{~cm}$, and collisions on the flight path will be negligible. After dissociation, $\mathrm{Hg}$ atoms must also exit the atomic beam environment, a distance of $<0.06 \mathrm{~cm}$. The density of $\mathrm{Hg}$ atoms in the beam is $n=2.4 \times 10^{11}$ atoms $\mathrm{cm}^{-3}$; thus, within the beam $\lambda_{m} \approx 400 \mathrm{~cm} \gg 0.06 \mathrm{~cm}$.

\section{SUMMARY}

We have described a definitive test of the previously untested strong Bell inequalities. It closes the detector efficiency loophole and provides for rigorous enforcement of Einstein locality. The experiment is clearly feasible. Furthermore, this proposed generation of an atomic entangled state has a feature that is quite different from previous experiments. Specifically, the lifetime of this entangled state is in the millisecond time regime as compared to lifetimes of a few nsec for existing experiments based on entangled states of photons.

\section{ACKNOWLEDGMENTS}

This research was supported by the Robert A. Welch Foundation Grant No. A-1218, the Texas Advanced Research Program Grant No. 010366-017, and by the National Science
Foundation, Grant No. PHY-9221038. The authors thank John Clauser and Marlan Scully for many helpful discussions, Jason Pan for his active participation, Joseph Eberly for suggesting the counterintuitive scheme, and Michael Fleischhauer and Markus Löffler for help with the master equation calculations.

\section{APPENDIX}

The quantum-mechanical predictions for tests of Bell inequalities are examined for arbitrary orientations of the detection planes. Consider a pair of spatially separated atoms each with spin $\frac{1}{2}$ in an entangled state with total spin zero; its state is given by Eq. (4). The spin state of each atom of the pair is measured in directions specified by the spherical coordinates $\left(\theta_{1}, \Phi_{1}\right)$ and $\left(\theta_{2}, \Phi_{2}\right)$, respectively, which are defined with respect to a fixed, arbitrarily oriented coordinate system. The quantum-mechanical prediction $R_{++}\left(\theta_{1}, \Phi_{1}, \theta_{2}, \Phi_{2}\right)$ is defined as the coincidence rate for observing both atoms in the spin-up state with respect to the directions defined by these spherical coordinates.

The operator for measuring the spin $S$ of a spin- $\frac{1}{2}$ particle in an arbitrary direction, defined by spherical coordinates $(\theta, \Phi)$, is given by

$$
S_{n}=S_{x} \sin \theta \cos \Phi+S_{y} \sin \theta \sin \Phi+S_{z} \cos \theta
$$

and can be written in matrix form with the help of the Paulispin matrices $\sigma_{x}, \sigma_{y}$, and $\sigma_{z}$,

$$
S_{n}=\frac{\hbar}{2}\left(\begin{array}{cc}
\cos \theta & \sin \theta e^{-i \Phi} \\
\sin \theta e^{i \Phi} & -\cos \theta
\end{array}\right) .
$$

The eigenvectors $\left|\chi_{\uparrow}\right\rangle,\left|\chi_{\downarrow}\right\rangle$ for this matrix and the corresponding eigenvalues $v_{\uparrow}, v_{\downarrow}$ can be readily found to yield [71]

$$
\begin{aligned}
& \left|\chi_{\uparrow}\right\rangle=\left(\begin{array}{c}
\cos \frac{\theta}{2} \\
\sin \frac{\theta}{2} e^{i \Phi}
\end{array}\right), \quad v_{\uparrow}=+\frac{\hbar}{2}, \\
& \left|\chi_{\downarrow}\right\rangle=\left(\begin{array}{c}
\sin \frac{\theta}{2} \\
-\cos \frac{\theta}{2} e^{i \Phi}
\end{array}\right), \quad v_{\downarrow}=-\frac{\hbar}{2} .
\end{aligned}
$$

The result for the quantum-mechanical expectation value of finding particle 1 in the state spin up with respect to an arbitrary direction, and finding atom 2 in the state spin up with respect to some other direction, is given by

$$
\begin{aligned}
R_{++} & \left(\theta_{1}, \Phi_{1}, \theta_{2}, \Phi_{2}\right) \\
& =\eta_{1} \eta_{2} f g N\left\langle\Psi \chi_{\uparrow 1}\right\rangle\left|\chi_{\uparrow 2}\right\rangle\left\langle\chi_{\uparrow 2}\right|\left\langle\chi_{\uparrow 1} \mid \Psi\right\rangle,
\end{aligned}
$$

where the parameters are the same as in Eq. (8). Substituting Eqs. (4), (A3), and (A4) into Eq. (A5), we get 


$$
\begin{aligned}
R_{++}\left(\theta_{1}, \Phi_{1}, \theta_{2}, \Phi_{2}\right)= & \frac{1}{2} \eta_{1} \eta_{2} f g N \mid \cos \frac{\theta_{1}}{2} \sin \frac{\theta_{2}}{2} e^{-i \Phi_{2}} \\
& -\left.\sin \frac{\theta_{1}}{2} \cos \frac{\theta_{2}}{2} e^{-i \Phi_{1}}\right|^{2},
\end{aligned}
$$

which can be rewritten

$$
\begin{aligned}
R_{++}\left(\theta_{1}, \Phi_{1}, \theta_{2}, \Phi_{2}\right)= & \frac{1}{2} \eta_{1} \eta_{2} f g N\left[\cos ^{2} \frac{\theta_{1}}{2} \sin ^{2} \frac{\theta_{2}}{2}\right. \\
& +\sin ^{2} \frac{\theta_{1}}{2} \cos ^{2} \frac{\theta_{2}}{2}-2 \cos \frac{\theta_{1}}{2} \sin \frac{\theta_{1}}{2} \\
& \left.\times \cos \frac{\theta_{2}}{2} \sin \frac{\theta_{2}}{2} \cos \left(\Phi_{1}-\Phi_{2}\right)\right]
\end{aligned}
$$

Thus in the general case for fixed detector planes, i.e., fixed values of $\Phi_{1}$ and $\Phi_{2}$, the quantum-mechanical prediction depends on $\theta_{1}$ and $\theta_{2}$ separately, not just their difference. Of course, for two directions $\left(\theta_{1}, \Phi_{1}\right)$ and $\left(\theta_{2}, \Phi_{2}\right)$, it is always possible to express $R_{++}$entirely in terms of the angle $\theta_{12}$ between these two directions; but this would be inconvenient in a test of Bell inequalities because the plane containing $\theta_{i j}$ would, in general, be different for every pair of directions $i, j$. Therefore we restrict consideration to a single coordinate system with fixed detection planes by $\Phi_{1}$ and $\Phi_{2}$.
For the case of parallel detection planes, as shown in Fig. $1, \Phi_{1}$ equals $\Phi_{2}$, and it is easy to show that Eq. (A6) reduces to our previous result, Eq. (11). In the coordinate system of Fig. 1, if the orientation of the detection planes is changed so that $\Phi_{1}=-\Phi_{2}=61.3^{\circ}$, then the detection planes would be perpendicular to the flight paths of the $\mathrm{Hg}$ fragments. In this case, evaluating the Bell inequality, Eq. (14), using Eq. (A7) with the same set of angles $\theta_{1}$ and $\theta_{2}$ as for parallel planes, Eq. (15), gives the quantum-mechanical prediction

$$
S_{\mathrm{QM}}\left(135^{\circ}, 0^{\circ}, 225^{\circ}, 90^{\circ}\right)=0.66 \eta g .
$$

This quantum-mechanical prediction cannot exceed 1.0 even when $\eta=g=1.0$. Hence, no violation of the Bell inequalities can be observed. It is possible, however, to find another set of angles for which the quantum-mechanical predictions do violate the Bell inequalities when $\Phi_{1}=-\Phi_{2}=61.3^{\circ}$, e.g.,

$$
S_{\mathrm{QM}}\left(208^{\circ}, 0^{\circ}, 151.7^{\circ}, 90^{\circ}\right)=1.07 \eta g \text {. }
$$

This result requires higher values of $\eta$ and $g$ than that for parallel detection planes, Eq. (15); in fact, the optimum arrangement is with parallel detection planes, as shown in Fig. 1. It should also be noted that for perpendicular detection planes, i.e., $\Phi_{1}-\Phi_{2}=90^{\circ}$, no set of angles can be found, so that the quantum-mechanical predictions violate the Bell inequality, Eq. (14).
[1] A. Einstein, B. Podolsky, and N. Rosen, Phys. Rev. 47, 777 (1935).

[2] J. S. Bell, Physics 1, 195 (1964).

[3] J. F. Clauser, M. A. Horne, A. Shimony, and R. A. Holt, Phys. Rev. Lett. 23, 880 (1969).

[4] J. F. Clauser and A. Shimony, Rep. Prog. Phys. 41, 1881 (1978).

[5] S. J. Freedman and J. F. Clauser, Phys. Rev. Lett. 28, 938 (1972).

[6] J. F. Clauser, Phys. Rev. Lett. 36, 1223 (1976).

[7] E. S. Fry and R. C. Thompson, Phys. Rev. Lett. 37, 465 (1976).

[8] E. S. Fry, Quantum Semiclass. Opt. 7, 229 (1995).

[9] A. Aspect, P. Grangier, and G. Roger, Phys. Rev. Lett. 49, 91 (1982).

[10] A. Aspect, J. Dalibard, and G. Roger, Phys. Rev. Lett. 49, 1804 (1982).

[11] C. O. Alley and Y. H. Shih, in Proceedings of the International Symposium Foundations of Quantum Mechanics, edited by M. Namiki (Physical Society of Japan, Tokyo, 1987), pp. 47-52.

[12] Z. Y. Ou and L. Mandel, Phys. Rev. Lett. 61, 50 (1988).

[13] J. G. Rarity and P. R. Tapster, Phys. Rev. Lett. 64, 2495 (1990).

[14] J. F. Clauser and M. A. Horne, Phys. Rev. D 10, 526 (1974).

[15] P. G. Kwiat, P. H. Eberhard, A. M. Steinberg, and R. Y. Chiao, Phys. Rev. A 49, 3209 (1994).

[16] E. S. Fry, in Proceedings of the International Conference on Lasers '92, edited by C. P. Wang (STS, Houston, TX, 1992), pp. $621-625$.
[17] E. Fry, in Third International Conference on Squeezed States and Uncertainty Relations, edited by D. Han, Y. S. Kim, N. H. Rubin, and W. W. Zachary, NASA Conf. Publ. No. 3270 (NASA, Baltimore, MD, 1993), pp. 575-580.

[18] R. D. v. Zee, S. C. Blankespoor, and T. S. Zwier, J. Chem. Phys. 88, 4650 (1988).

[19] A. Zehnacker, M. C. Duval, C. Jouvet, C. Lardeux-Dedonder, D. Solgadi, B. Soep, and O. B. d'Azy, J. Chem. Phys. 86, 6565 (1987).

[20] C. Brechignac, M. Broyer, P. Cahuzac, G. Delacretaz, P. Labastie, J. P. Wolf, and L. Wöste, Phys. Rev. Lett. 60, 275 (1988).

[21] S. H. Linn, C. L. Liao, C. X. Liao, J. J. M. Brom, and C. Y. Ng, Chem. Phys. Lett. 105, 645 (1984).

[22] K. Rademann, B. Kaiser, U. Even, and F. Hensel, Phys. Rev. Lett. 59, 2319 (1987).

[23] C. Y. Ng (private communication).

[24] T. S. Zwier (private communication).

[25] D. R. Miller, in Atomic and Molecular Beam Methods, edited by G. Scoles (Oxford University Press, New York, 1988), pp. 14-53.

[26] W. R. Gentry, in Atomic and Molecular Beam Methods, edited by G. Scoles (Oxford University Press, New York, 1988), pp. 54-82.

[27] D. Bahat, O. Cheshnovsky, U. Even, N. Lavie, and Y. Magen, J. Phys. Chem. 91, 2460 (1987).

[28] U. Even, N. Ben-Horin, and J. Jortner, Chem. Phys. Lett. 156, 138 (1989). 
[29] G. Herzberg, Molecular Spectra and Molecular Structure (Van Nostrand, Princeton, NJ, 1950), pp. 275-276.

[30] E. W. Smith, R. E. Drullinger, M. M. Hessel, and J. Cooper, J. Chem. Phys. 66, 5667 (1977).

[31] M. Stock, E. W. Smith, R. E. Drullinger, M. M. Hessel, and J. Pourcin, J. Chem. Phys. 68, 1785 (1978).

[32] J. Tellinghuisen, J. Mol. Spectrosc. 103, 455 (1984).

[33] J. Tellinghuisen, Adv. Chem. Phys. 60, 299 (1985).

[34] G. Herzberg, Molecular Spectra and Molecular Structure (Van Nostrand, Princeton, NJ, 1950), pp. 141-145.

[35] T. K. Lo, Ph.D. thesis, Boston University, 1980 (unpublished).

[36] W. Demtröder, in Laser Spectroscopy, edited by F. P. Schäfer, Springer Series in Chemical Physics Vol. 5 (Springer-Verlag, New York, 1981), pp. 15 and 59.

[37] E. C. Benck, J. E. Lawler, and J. T. Dakin, J. Opt. Soc. Am. B 6, 11 (1989).

[38] F. Bitter, Appl. Opt. 1, 1 (1962).

[39] B. Cheron, J. L. Cojan, J. Landais, and M. Aymar, J. Phys. B 22, 2129 (1989).

[40] J. E. Field (private communication).

[41] J. Berkowitz, Photoabsorption, Photoionization, and Photoelectron Spectroscopy (Academic, New York, 1979).

[42] J. Berkowitz and C. Lifshitz, J. Phys. B 1, 438 (1968).

[43] W. Demtröder, Laser Spectroscopy (Ref. [36]), p. 34, Eqs. 2.66 and 2.69 .

[44] U. Fano and J. W. Cooper, Phys. Rev. 137, A1364 (1965).

[45] R. Lincke and B. Stredele, Z. Phys. 238, 164 (1970).

[46] R. N. Zare, Angular Momentum (Wiley, New York, 1988), p. 39.

[47] K. Miyazaki, T. Watanabe, and K. Fukuda, J. Phys. Soc. Jpn. 40, 233 (1976).

[48] W. Demtröder, Laser Spectroscopy, (Ref. [36]), p. 33, Eq. 2.61 .

[49] V. S. Letokhov, Laser Photoionization Spectroscopy (Academic, Orlando, FL, 1987), p. 53.

[50] S. Li and F. Lin, J. Phys. B 22, 1183 (1989).

[51] W. Demtröder, Laser Spectroscopy (Ref. [36]), pp. 37 and 59.
[52] A. C. G. Mitchell and M. W. Zemansky, Resonance Radiation and Excited Atoms (Cambridge University Press, New York, 1961), p. 97, Eq. 31.

[53] J. Oreg, F. T. Hioe, and J. H. Eberly, Phys. Rev. A 29, 690 (1984).

[54] U. Gaubatz, P. Rudecki, S. Schiemann, and K. Bergmann, J. Chem. Phys. 92, 5363 (1990).

[55] B. A. Bushaw, Anal. Chem. 57, 2397 (1985).

[56] P. Dyer, G. C. Baldwin, C. Kittrell, D. G. Imre, and E. Abramson, Appl. Phys. Lett. 42, 311 (1983).

[57] P. Dyer, G. C. Baldwin, A. M. Sabbas, C. Kittrell, E. L. Schweitzer, E. Abramson, and D. G. Imre, J. Appl. Phys. 58, 2431 (1985).

[58] W. L. Glab and J. P. Hessler, Appl. Opt. 26, 3181 (1987).

[59] P. Lokai, B. Burghardt, and W. Mückenheim, Appl. Phys. B 45, 245 (1988).

[60] W. Mückenheim, P. Lokai, B. Burghardt, and D. Basting, Appl. Phys. B 45, 259 (1988).

[61] K. Kato, IEEE J. Quantum Electron. QE-22, 1013 (1986).

[62] G. Paschmann, Rev. Sci. Instrum. 41, 1706 (1970).

[63] K. C. Schmidt, The Channeltron Electron Multiplier Model CEM 4010, Application Note 9803, Bendix Electro-Optics Division, 1969.

[64] S. A. Fields, J. L. Burch, and W. A. Oran, Rev. Sci. Instrum. 48, 1076 (1977).

[65] E. P. Wentworth and K. Mauersberger, Rev. Sci. Instrum. 43, 1327 (1972).

[66] K. H. Krebs, Vacuum 33, 7 (1983).

[67] J. Richards, J. Phys. E 17, 1081 (1984).

[68] A. Guthrie, Vacuum Technology (Wiley, New York, 1963), p. 135.

[69] E. S. Fry, Phys. Rev. A 8, 1219 (1973).

[70] A. A. Radzig and B. M. Smirnov, Reference Data on Atoms, Molecules, and Ions, edited by V. I. Goldanskii, R. Gomer, F. P. Schäfer, and J. P. Toennies, Springer Series in Chemical Physics Vol. 31 (Springer-Verlag, New York, 1980).

[71] B. H. Bransden and C. J. Joachain, Introduction to Quantum Mechanics (Wiley, New York, 1992), pp. 290-292. 